\title{
Invariants of Newton non-degenerate surface singularities
}

\author{
Gábor Braun and András Némethi \\ Dedicated to J. Steenbrink on his 60th birthday
}

\begin{abstract}
We recover the Newton diagram (modulo a natural ambiguity) from the link for any surface hypersurface singularity with non-degenerate Newton principal part whose link is a rational homology sphere. As a corollary, we show that the link determines the embedded topological type, the Milnor fibration, and the multiplicity of such a germ. This proves (an even stronger version of) Zariski's conjecture about the multiplicity for such a singularity.
\end{abstract}

\section{Introduction}

In general, it is a rather challenging task to connect the analytic and topological invariants of normal surface singularities. The program which aims to recover different discrete analytic invariants from the abstract topological type of the singularity (i.e. from the oriented homeomorphism type of the link $K$, or from the resolution graph) can be considered as a continuation of the work of Artin, Laufer, Tomari, Yau, and the second author about rational and elliptic singularities. It includes the efforts of Neumann and Wahl to recover the possible equations of the universal abelian covers [NW02], and the efforts of the second author and Nicolaescu about the possible connections of the geometric genus with the Seiberg-Witten invariants of the link [NN02]. See [Ném04] for a review of this program.

In order to have a chance for this program, one has to consider a topological restriction (the weakest restriction for which we still hope for positive results may be that the link is a rational homology sphere) and, in addition, a restriction about the analytic type of the singularity. By [LMN05], the Gorenstein condition is not sufficient. We expect pathologies even for hypersurface singularities.

For isolated hypersurface singularities, a famous conjecture was formulated by Zariski [Zar71], which predicts that the multiplicity is determined by the embedded topological type. For hypersurface germs with rational homology sphere links, Mendris and the second author in [MN05] formulated (and verified for suspension singularities) an even stronger conjecture, namely that the abstract link already determines the embedded topological type, the multiplicity, and equivariant Hodge numbers (of the vanishing cohomology).

The goal of the present article is to verify this stronger conjecture for isolated singularities with non-degenerate Newton principal part. In fact, we will prove that from the link (provided that it is a rational homology sphere) one can recover the Newton boundary (up to a natural ambiguity, see

Received 13 July 2006, accepted in final form 25 January 2007.

2000 Mathematics Subject Classification 14J17, 14Q10 (primary), 52B20 (secondary).

Keywords: hypersurface singularities, links of singularities, resolution graphs, Newton boundary, Newton polyhedrons.

The first author is partially supported by Hungarian National Research Fund, grant No. T 042 769. The second author is partially supported by NSF grant DMS-0304759, Marie Curie and OTKA grants.

This journal is (C) Foundation Compositio Mathematica 2007. 


\section{G. Braun AND A. NÉmethi}

Theorem 1.0.1, and up to a permutation of coordinates), and hence the equation of the germ (up to an equisingular deformation). This is the most that we can hope for.

The reader is invited to consult [AGV88, Ném04] for general facts about singularities. Section 2 reviews the terminology and some properties of germs with non-degenerate Newton principal part. In $\S 3$ we define the equivalence relation $\sim$ of Newton boundaries characterizing the above-mentioned ambiguities. It may also be generated by the following elementary step: two diagrams $\Gamma_{1}$ and $\Gamma_{2}$ are equivalent if both define isolated singularities and $\Gamma_{1} \subset \Gamma_{2}$. (At the level of germs, this can be described by a linear deformation.) Although the structure of an equivalence class is not immediate from the definition, we define an easily recognizable representative in every class, which we call the d-minimal representative.

In $\S 4.2$ we review Oka's algorithm which provides a possible resolution graph $G(\Gamma)$ (or, equivalently, a plumbing graph of the link) from the Newton boundary $\Gamma$ (see [Oka87]). (Equivalent graphs provide plumbing graphs related by blowing ups/downs, and hence determine the same link.) Our main result says that Oka's algorithm can be essentially inverted.

Theorem 1.0.1. Assume that the Newton diagrams $\Gamma_{1}$ and $\Gamma_{2}$ determine isolated singularities with non-degenerate Newton principal part whose links are rational homology spheres. Assume that the good minimal resolution graphs associated with $G\left(\Gamma_{1}\right)$ and $G\left(\Gamma_{2}\right)$ are isomorphic. Then (up to a permutation of coordinates) $\Gamma_{1} \sim \Gamma_{2}$. In particular, from the link $K$, one can identify the $\sim$-equivalence class of the Newton boundary (up to a permutation of coordinates) or, equivalently, the d-minimal representative of this class.

In fact, we prove an even stronger result: one can already recover the corresponding class of Newton diagrams (or its distinguished representative) from the orbifold diagram $G^{o}$ associated with the good minimal resolution graph. This diagram, a priori, contains less information than the resolution graph, because it codifies only its shape and some subgraph determinants, see $\S 4.4$ for details. (Although $G^{o}$ has a different decoration, it is comparable with the 'splice diagram' considered in [NW02].)

As most of the invariants of the germs are stable under the deformations defining the equivalence relation $\sim($ see $\S 3.2)$, one has the following.

Corollary 1.0.2. Let $f$ be an isolated germ with non-degenerate Newton principal part whose link is a rational homology sphere. Then the oriented topological type of its link determines completely its Milnor number, geometric genus, spectral numbers, multiplicity, and, finally, its embedded topological type.

Such a statement is highly non-trivial for any of the above invariants. For the history of the problem regarding the Milnor number and the geometric genus, the reader is invited to consult [Ném04]. Here we emphasize only the following.

(i) Regarding the embedded topological type, Corollary 1.0.2 shows that if a rational homology sphere 3-manifold can be embedded into $S^{5}$ as the embedded link of an isolated hypersurface singularity with non-degenerate Newton principal part, then this embedding is unique. (Note the huge difference to the case of plane curves, and also to the higher-dimensional case, where already the Brieskorn singularities provide a large variety of embeddings $S^{2 n-1} \subset S^{2 n+1}$, $n \neq 2$.)

(ii) Such a link can be realized by a germ $f$ with non-degenerate Newton principal part in an essentially unique way, i.e. up to a sequence of linear $\mu$-constant deformations (corresponding to $\sim$ ) and permutation of coordinates, see Corollary 5.1.3(ii).

Regarding the main theorem, some more comments are in order. 


\section{INVARIANTS OF NEWTON NON-DEGENERATE SURFACE SINGULARITIES}

(a) The assumption that the link is a rational homology sphere is necessary: the germs $\left\{z_{1}^{a}+z_{2}^{b}+\right.$ $\left.z_{3}^{c}=0\right\}$ with exponents $(3,7,21)$ and $(4,5,20)$ share the same minimal resolution graph.

(b) The proof of Theorem 1.0.1 is, in fact, a constructive algorithm which provides the d-minimal representatives of the corresponding class of diagrams from the orbifold diagram $G^{o}$.

Hence, one may check effectively whether an arbitrary resolution graph can be realized by a hypersurface singularity with non-degenerate Newton principal part. Indeed, if one runs our algorithm and it fails, then it is definitely not of this type. If the algorithm goes through and provides some candidate for a Newton diagram, then one has to compute the graph (orbifold diagram) of this candidate (by Oka's procedure) and compare it with the initial one. If they agree, then the answer is yes; if they are different, the answer is again no (this may happen because our algorithm uses only a part of the information of $G^{\circ}$ ).

For example, one can check that the resolution graph shown in Figure 1 cannot be realized by an isolated singularity with non-degenerate Newton principal part (although it can be realized by a suspension $\left\{z_{3}^{2}+g\left(z_{1}, z_{2}\right)=0\right\}$, where $g$ is an irreducible plane curve singularity with Newton pairs $(2,3)$ and $(1,3))$.

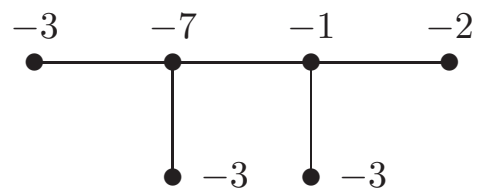

FigURE 1. A resolution graph not coming from a singularity with non-degenerate Newton principal part.

We mention that, in general, there is no procedure to decide whether a graph is the resolution graph of a hypersurface isolated singularity (this is one of the open problems asked by Laufer [HR89, p. 122]; for suspension singularities, it is solved in [MN05]).

\section{Singularities with non-degenerate Newton principal part}

\subsection{The Newton boundary and criterion for isolated singularities}

2.1.1 For any set $S \subset \mathbb{N}^{3}$ denote by $\Gamma_{+}(S) \subset \mathbb{R}^{3}$ the convex closure of $\bigcup_{p \in S}\left(p+\mathbb{R}_{+}^{3}\right)$. We call the 1-faces of any polytope edges, and face will simply mean a 2-face. The collection of all boundary faces of $\Gamma_{+}(S)$ is denoted by $\mathcal{F}$. The set of compact faces of $\Gamma_{+}(S)$ is denoted by $\mathcal{F}_{c}$. By definition, the Newton boundary (or diagram) $\Gamma(S)$ associated with $S$ is the union of compact boundary faces of $\Gamma_{+}(S)$. Let $\partial \Gamma$ denote the union of those edges of $\Gamma(S)$ which are not the intersection of two faces of $\Gamma(S)$. Let $\Gamma_{-}(S)$ denote the cone with base $\Gamma(S)$ and vertex 0 .

Let $f:\left(\mathbb{C}^{3}, 0\right) \rightarrow(\mathbb{C}, 0)$ be an analytic function germ defined by a convergent power series $\sum_{p} a_{p} z^{p}$ (where $p=\left(p_{1}, p_{2}, p_{3}\right)$ and $z^{p}=z_{1}^{p_{1}} z_{2}^{p_{2}} z_{3}^{p_{3}}$ ). By definition, the Newton boundary $\Gamma(f)$ of $f$ is $\Gamma(\operatorname{supp}(f))$, where $\operatorname{supp}(f)$ is the support $\left\{p: a_{p} \neq 0\right\}$ of $f$, and we write $\Gamma_{-}(f)$ for $\Gamma_{-}(\operatorname{supp}(f))$. The Newton principal part of $f$ is $\sum_{p \in \Gamma(f)} a_{p} z^{p}$. Similarly, for any $q$-face $\triangle$ of $\Gamma(f)$ (of any dimension $q$ ), set $f_{\triangle}(z):=\sum_{p \in \triangle} a_{p} z^{p}$. We say that $f$ is non-degenerate on $\triangle$ if the system of equations $\partial f_{\triangle} / \partial z_{1}=\partial f_{\triangle} / \partial z_{2}=\partial f_{\triangle} / \partial z_{3}=0$ has no solution in $\left(\mathbb{C}^{*}\right)^{3}$. When $f$ is non-degenerate on every $q$-face of $\Gamma(f)$, we say (after Kouchnirenko [Kou76]) that $f$ has a non-degenerate Newton principal part. The diagram $\Gamma(f)$ and the function $f$ are called convenient if $\Gamma(f)$ intersects all of the coordinate axes. 


\section{G. Braun AND A. NÉmethi}

2.1.2 In this article we assume that $f$ is singular, i.e. $\partial f(0)=0$.

2.1.3 If we fix a Newton boundary $\Gamma$ (i.e. $\Gamma=\Gamma(S)$ for some $S$ ), then the set of coefficients $\left\{a_{p}: p \in \Gamma\right\}$ for which $f(z)=\sum_{p \in \Gamma} a_{p} z^{p}$ is Newton non-degenerate (as its own principal part) form a non-empty Zariski open set (cf. [Kou76, Theorem 1.10(iii)]). Nevertheless, even for generic coefficients $\left\{a_{p}\right\}_{p \in \Gamma}$, the germ $f=\sum_{p \in \Gamma} a_{p} z^{p}$ (or any $f$ with $\Gamma(f)=\Gamma$ ), in general, does not define an isolated singularity. The germ $f$ (with generic $\left\{a_{p}\right\}_{p \in \Gamma}$ ) defines an isolated singularity if and only if $\Gamma$ satisfies the following additional properties (see [Kou76, Remark 1.13(ii)]):

(i) $\{(0,0,0),(0,0,1),(0,1,0),(1,0,0)\} \cap \Gamma=\emptyset$ (cf. (2.1.2));

(ii) the diagram $\Gamma$ has a vertex on every coordinate plane; and

(iii) for every coordinate axis, $\Gamma$ has a vertex at a distance of at most 1 from the axis.

For example, a convenient $f$ with generic coefficients defines an isolated singularity.

Example 2.1.4. Note that (2.1) cannot be satisfied by one vertex. Moreover, if $\Gamma$ satisfies (2.1) and has no faces, then (modulo a permutation of the coordinates) it is the segment $[(0,1,1),(n, 0,0)]$ for some $n \geqslant 2$.

Remark 2.1.5. Assume that $\Gamma$ is not an edge. Then (2.1) implies that every edge of $\partial \Gamma$ should lie either on a coordinate plane or be (after permuting coordinates) of the form $A B=[(a, 0, c),(0,1, b)]$ with $a>0$ and $b+c>0$. The number of edges of second type coincides with the number of coordinate axes not intersected by $\Gamma$. (Indeed, assume that the $z_{3}$ axis does not meet $\Gamma$. Project $\Gamma$ to the $z_{1} z_{2}$ plane by $\psi\left(z_{1}, z_{2}, z_{3}\right)=\left(z_{1}, z_{2}\right)$. Then, by $(2.1)$, the boundary of $\psi(\Gamma)$ contains an edge of type $[(a, 0),(0,1)]$.

2.1.6 If one tries to analyze the invariants of a germ in terms of its Newton diagram (see, e.g., the references cited in $\S 2.2$ ), one inevitably faces the arithmetical properties of integral polytopes. In Appendix A.1, we collect those which will be used in the body of the paper. The relevant notation and terminologies are listed in the following.

Notation/Definitions 2.1.7. Fix a Newton diagram. Set $\triangle \in \mathcal{F}$. Let $\nabla \in \mathcal{F}$ be an adjacent face with a common (compact) edge $A B:=\triangle \cap \nabla$. Then one defines:

(i) $\overrightarrow{\mathbf{a}}_{\triangle}$, the normal vector of $\triangle$, i.e. the primitive integral vector with non-negative entries, normal to $\triangle$;

(ii) $t_{\triangle, \nabla}$, the number of components of $A B \backslash \mathbb{N}_{>0}^{3}$;

(iii) $n_{\triangle, \nabla}$, the determinant of $\overrightarrow{\mathbf{a}} \triangle$ and $\overrightarrow{\mathbf{a}}_{\nabla}$, namely, the greatest common divisor of the entries of the cross product $\overrightarrow{\mathbf{a}} \triangle \times \overrightarrow{\mathbf{a}}_{\nabla}\left(n_{\triangle, \nabla} \geqslant 1\right)$;

(iv) $\overrightarrow{\mathbf{e}_{\mathbf{1}}}, \overrightarrow{\mathbf{e}_{\mathbf{2}}}, \overrightarrow{\mathbf{e}_{3}}$, the three coordinate normal vectors.

The number $n_{\triangle, \nabla}$ is also called the determinant of the edge $A B$. As it depends only on the corresponding normal vectors, sometimes we put the normal vectors in the index instead of the faces. For example, if $\overrightarrow{\mathbf{a}}_{\nabla}=\overrightarrow{\mathbf{e}_{\mathbf{i}}}$ and $\triangle \in \mathcal{F}_{c}$, then we may also write $n_{\triangle, \overrightarrow{\mathbf{e}_{\mathbf{i}}}}$ for $n_{\triangle, \nabla}$. The number $t_{\triangle, \overrightarrow{\mathbf{e}_{\mathbf{i}}}}$ has a similar meaning. In fact, with the notation $\overrightarrow{\mathbf{a}}_{\triangle}=\left(a_{1}, a_{2}, a_{3}\right)$, one has

$$
n_{\triangle, \overrightarrow{\mathbf{e}_{\mathbf{i}}}}=\operatorname{gcd}\left(a_{j}, a_{k}\right) \text {, where }\{i, j, k\}=\{1,2,3\} .
$$

Similarly, for any lattice polygon $\triangle$, the vector $\overrightarrow{\mathbf{a}} \triangle$ denotes the primitive integral vector normal to $\triangle$ (well-defined up to a sign). The combinatorial area, by definition (cf. [Oka87, (6.2)]), is

$$
g(\triangle):=2 \#\{\text { inner lattice points }\}+\#\{\text { border lattice points }\}-2 .
$$

Clearly, $g(\triangle)$ is additive. The face $\triangle$ is called empty if its only lattice points are its vertices. 


\section{INVARIANTS OF NEWTON NON-DEGENERATE SURFACE SINGULARITIES}

\subsection{Some discrete invariants determined from the Newton boundary}

If $f$ defines an isolated singularity and has a non-degenerate Newton principal part, then its Newton boundary $\Gamma(f)$ determines almost all of its discrete analytic and embedded topological invariants. We list some examples.

(a) The Milnor number $\mu(f)$ of $f$ is given by Kouchnirenko [Kou76]. For any $\Gamma$ let $V_{3}$ be the threedimensional volume of $\Gamma_{-}$, and for $1 \leqslant q \leqslant 2$, let $V_{q}$ be the sum of the $q$-dimensional volumes of all of the intersections of $\Gamma$ - with $q$-dimensional coordinate planes. Set $\nu(\Gamma):=6 V_{3}-2 V_{2}+V_{1}-1$. Then, by [Kou76], the Milnor number $\mu(f)$ of any convenient germ $f$ with non-degenerate Newton principal part is given combinatorially via $\Gamma(f)$ by

$$
\mu(f)=\nu(\Gamma(f)) .
$$

In fact, the same formula is also valid for non-convenient isolated singularities. Indeed, assume, for example, that the diagram $\Gamma(f)$ does not intersect the $z_{3}$ axis, and let $A B$ be an edge as in Remark 2.1.5. Then the deformation $f_{d}:=f+t z_{3}^{d}$ with $d \geqslant \mu(f)+2$ has a uniform stable radius for the Milnor fibration [Oka79], hence $\mu(f)=\mu\left(f_{d}\right)$. Moreover, $\Gamma_{-}\left(f_{d}\right)=\Gamma_{-}(f) \cup W_{d}$, where $W_{d}$ is the 3 -simplex with vertices $0, A, B$ and $(0,0, d)$. As $\left(6 V_{3}-2 V_{2}+V_{1}\right)\left(W_{d}\right)=0$, one gets that $\nu\left(\Gamma\left(f_{d}\right)\right)=\nu(\Gamma(f))$.

(Since $f$ is finitely determined, $f$ and $f_{d}$ are right-equivalent for $d \gg 0$ and their other invariants listed in this section also agree. Hence, being convenient, present in many cases in the literature, is not really essential for us; see also [Wal99].)

(b) The characteristic polynomial of the algebraic monodromy is determined in [Var76]. The geometric genus of the surface singularity $(\{f=0\}, 0)$ is given by $\#\left(\Gamma_{-}(f) \cap \mathbb{N}_{>0}^{3}\right)$, cf. [MT80, Sai88]. The set of spectral numbers (or characteristic exponents) is computed in [Dan79, Sai88, Ste77, VK85]. The multiplicity of $f$ is given by $\min _{p \in \Gamma(f)} \sum p_{i}$.

(c) The embedded topological type and the Milnor fibration of $f$ (with its homological 'package' including the Seifert form) is determined from $\Gamma(f)$ uniquely by [Oka79, 2.1].

(d) An explicit construction of the dual resolution graph $G(f)$ of the surface singularity $(\{f=0\}, 0)$ is given in [Oka87] (we review this in $\S 4.2$ ).

\subsection{The structure of Newton polytopes in the case of rational homology sphere links}

2.3.1 An important assumption of the main result of the present article is that the link $K(f)$ of $f$ is a rational homology sphere, i.e. $H_{1}(K(f), \mathbb{Q})=0$. This additional assumption (in addition to $(2.1)$, which says that $f$ with non-degenerate Newton principal part is an isolated singularity) imposes serious restrictions on the Newton boundary $\Gamma(f)$, cf. [Sai88]:

$$
K(f) \text { is a rational homology sphere } \Longleftrightarrow \Gamma(f) \cap \mathbb{N}_{>0}^{3}=\emptyset .
$$

In this section we assume that $\Gamma(f)$ satisfies these two restrictions, namely (2.1) and (2.5). Our goal is to derive the structure theorem (Proposition 2.3.9) for Newton diagrams.

We fix a diagram $\Gamma$. We start by classifying the non-triangular faces.

Lemma 2.3.2. If a face of $\Gamma$ is not a triangle, then it is a trapezoid. By permuting coordinates, its vertices are $A=(p, 0, n), B=(0, q, n), C=\left(r_{1}, r_{2}+t q, 0\right)$ and $D=\left(r_{1}+t p, r_{2}, 0\right)$, where $p, q>0$, $\operatorname{gcd}(p, q)=1, t \geqslant 1$ and $r_{1}, r_{2} \geqslant 0$. The only side which can have inner lattice points is the base lying on the $z_{1} z_{2}$ plane (with $t-1$ of them).

Proof. The idea of the proof is the following: if a lattice polygon $\triangle$ is not a triangle or a trapezoid, then there exists a parallelogram in $\triangle$ with three vertices on the boundary of $\triangle$ and one in its interior, which contradicts (2.5). The details are left to the reader. 


\section{G. Braun AND A. NÉmethi}

Terminology 2.3.3. The edges of a trapezoid have asymmetric roles. For future reference we give names to them. The bottom edge always lies on a coordinate plane. If two (or more) edges lie on coordinate planes, the bottom edge is that which has internal lattice points, if such exists. Otherwise, we choose one of them arbitrarily.

Opposite to the bottom edge lies the top edge, and the others are called side edges.

Terminology/Discussion 2.3.4. An edge crosses, say, the $z_{3}$ axis if it is of the form $[(p, 0, a),(0, q, b)]$, where $p>0, q>0$, and $a+b>0$. There are two types of edges on $\Gamma$ : those lying on a coordinate plane and those crossing a coordinate axis.

While edges of the first type do not 'cut' $\Gamma$, edges of the second type usually cut $\Gamma$ into two nonempty parts, one of which has a particularly simple structure. In order to see this, project $\mathbb{R}_{\geqslant 0}^{3} \backslash 0$ from the origin to the triangle $\mathbb{T}:=\left\{z_{1}+z_{2}+z_{3}=1: z_{i} \geqslant 0(i=1,2,3)\right\}$. The restriction $\phi: \Gamma \rightarrow \mathbb{T}$ is one-to-one and preserves segments. An edge lying on a coordinate plane projects into $\partial \mathbb{T}$, while a crossing edge projects into a segment with only its end points on $\partial \mathbb{T}$ and cutting $\mathbb{T}$ into two parts such that at least one of them, say $\mathbb{T}_{0}$, is a triangle. By Lemma 2.3.2, the projection of a trapezoid hits the interior of all of the sides of $\mathbb{T}$, hence $\phi^{-1}\left(\mathbb{T}_{0}\right)$ may contain only a 'sequence of triangles'. Therefore, one has the following.

Lemma 2.3.5. An edge of $\Gamma$ crossing (say) the $z_{3}$ axis, which is not on $\partial \Gamma$, cuts $\Gamma$ into two nonempty parts. Consider the plane $\pi$ formed by the edge and the origin. Then that part of $\Gamma$, which is on the same side of $\pi$ as the positive $z_{3}$ axis, consists only of triangular faces with vertices lying on the $z_{1} z_{3}$ and $z_{2} z_{3}$ planes. They form a sequence $\triangle_{1}, \ldots, \triangle_{k}$, where $\triangle_{i}$ is adjacent with $\triangle_{i+1}$ (and these are the only adjacent relations).

Corollary/Definition 2.3.6. Fix a coordinate axis.

First, assume that there is at least one triangular face whose vertices are on the two coordinate planes adjacent to the axis. Then the collection of such triangular faces form a sequence as in Lemma 2.3.5, and their union is called the arm of the diagram in the direction of that axis. The arm also contains all of the crossing edges whose vertices lie on the two coordinate planes. Let the hand be the triangle of the arm which is nearest to the axis (in the $\phi$-projection, say). Let the shoulder be the crossing edge of the arm which is most distant from the axis (in the same sense).

Next, assume that there is no triangular face whose vertices are on these two coordinate planes. Then we distinguish two cases.

(a) If there exists a crossing edge of the coordinate axis, then it is unique; in this case we say that the arm in that direction is degenerate, and the degenerate arm (and also its shoulder) is this unique crossing edge.

(b) If there is no crossing edge either, then we say that there is no arm in the direction of the axis.

Terminology 2.3.7. A triangular face of $\Gamma$ is called central if its vertices are not situated on the union of two coordinate planes. A face of $\Gamma$ is called central if it either is a central triangle or it is a trapezoid. An edge of $\Gamma$ is central if (modulo a permutation of the coordinates) it has the form $[(0,0, a),(p, q, 0)]$.

Using the projection $\phi: \Gamma \rightarrow \mathbb{T}$, one may easily verify the following.

Lemma 2.3.8. The diagram $\Gamma$ has at most one central face; $\Gamma$ has a central face if and only if it has no central edge.

These facts can be summarized in the following result on the structure of Newton diagrams.

Proposition 2.3.9. Every Newton diagram $\Gamma$ (which satisfies (2.1) and (2.5)) sits in exactly one of the three disjoint families characterized as follows. 


\section{INVARIANTS OF NEWTON NON-DEGENERATE SURFACE SINGULARITIES}

(1) The diagram $\Gamma$ has a unique central trapezoid with at most three disjoint (possibly degenerate) arms. The arms correspond to those sides of the trapezoid which are crossing edges.

(2) The diagram $\Gamma$ has a unique central triangle with three disjoint (possibly degenerate) arms.

(3) The diagram $\Gamma$ has (at least one) central edge.

Moreover, if $\Gamma$ has a central edge, then there are two cases. If $\Gamma$ has only one face, this face is triangular with all vertices on coordinate axes, then all edges are central. Otherwise, all central edges have a common intersection point (say $P$ ) sitting on a coordinate axis, and the diagram has two (possibly degenerate) arms in the direction of the other two axes. The arms may overlap each other, i.e. have common triangles. The point $P$ is a vertex of all of the triangles in the intersection of the arms, and all of those edges of the triangles which contain $P$ are central (and these are all of the central edges).

\section{Equivalent Newton boundaries: deformations}

\subsection{The equivalence relation}

3.1.1 Our aim is to recover the Newton boundary (up to a permutation of coordinates) of an isolated singularity with non-degenerate Newton principal part from the link $K(f)$, provided that $K(f)$ is a rational homology sphere. Strictly speaking, this is not possible: one can easily construct pairs of such germs having identical links but different boundaries. For example, take an isolated non-convenient germ $f$ and $f_{d}=f+\sum z_{i}^{d}$ with $d \gg 0$. This motivates the definition of a natural equivalence relation of Newton boundaries. By definition, it will be generated by two combinatorial 'steps'.

3.1.2 Fix a Newton boundary $\Gamma=\Gamma(S)$ which satisfies (2.1). Let $A B$ be an edge of $\partial \Gamma$ which is not contained in any coordinate plane. By Remark 2.1.5, up to a permutation of coordinates, $A=(a, 0, c)$ (with $a>0)$ and $B=(0,1, b)$.

Move 1. We add a new vertex $C=\left(a^{\prime}, 0, c^{\prime}\right)$ to $\Gamma$ in such a way that $\Gamma(S \cup C)=\Gamma(S) \cup \triangle_{A B C}$. Here $\triangle_{A B C}$, the 2-simplex spanned by the points $A, B, C$ appears as a new face. (In particular, $0 \leqslant a^{\prime}<a$ and $c^{\prime}$ must be sufficiently large.)

Move 2. Assume that $A B$ is in the face $\triangle$ whose supporting plane is $H$. The line through $A B$ cuts out the open semi-plane $H_{+}$of $H$ which does not contain $\triangle$. Set $S^{\prime}:=H_{+} \cap \mathbb{N}^{3}$. Then by adding a non-empty subset $S^{\prime \prime}$ of $S^{\prime}$ to $S$, we create a new Newton boundary $\Gamma\left(S \cup S^{\prime \prime}\right)$. By this move, all faces of $\Gamma(S)$ are unmodified, except $\triangle$, which is replaced by a larger face containing $\triangle$.

Definition 3.1.3. We denote Move 1 and Move 2 by $\mathrm{M} 1_{+}$and $\mathrm{M} 2_{+}$, respectively. We denote their inverses by $\mathrm{M} 1_{-}$and $\mathrm{M} 2_{-}$, respectively. The segment $A B$ will be called the axis of the corresponding move.

Two Newton diagrams $\Gamma_{1}$ and $\Gamma_{2}$, both satisfying (2.1), are equivalent (and we write $\Gamma_{1} \sim \Gamma_{2}$ ), if they can be connected by a sequence of elementary moves $\left(\mathrm{M} 1_{ \pm}\right.$or $\left.\mathrm{M} 2_{ \pm}\right)$, such that all of the intermediate Newton boundaries satisfy (2.1) as well.

Example 3.1.4. Using Remark 2.1.5 and induction, one can show that if $\Gamma_{1}$ and $\Gamma_{2}$ are Newton diagrams, $\Gamma_{1} \subset \Gamma_{2}$, both satisfying (2.1), then they are equivalent. In fact, the inclusion of Newton boundaries with (2.1) generates the same equivalence relation.

Example 3.1.5. The segments $[(0,1,1),(n, 0,0)]$ and $[(1,0,1),(0, n, 0)]$ (considered as diagrams) are equivalent. Indeed, add to $\Gamma_{1}=[(0,1,1),(n, 0,0)]$ the vertex $(1,0,1)$ (by $\left.\mathrm{M} 1_{+}\right)$, then add $(0, n, 0)$ (by $\mathrm{M} 2_{+}$), then remove the end points of $\Gamma_{1}$ (cf. Example 3.1.4). 


\section{G. Braun AND A. NÉmethi}

3.1.6 Sometimes it is more convenient to specify the deformation of the corresponding germs instead of the modification of Newton diagrams: adding a new vertex $p$ to $S$ translates into adding a new monomial $t a_{p} z^{p}$ to $f$, with $t \in[0, \epsilon]$ a deformation parameter. (The fact that these deformations are linear in $t$ is crucial in the proof of Proposition 3.2.1(c)).

Example 3.1.7. The number of 'essential' deformation parameters can be as large as we wish. For example, for $m, n \gg 0$, all of the different Newton diagrams associated with the family

$$
z_{3}\left(z_{1}^{p}+z_{2}^{q}+z_{3}^{r}\right)+\sum_{i} t_{i} z_{1}^{m-i p} z_{2}^{n+i q} \quad(m-i p \geqslant 0, n+i q \geqslant 0)
$$

satisfy (2.1), and are equivalent (via repeated $\mathrm{M} 2_{ \pm}$) as soon as $\sum_{i}\left|t_{i}\right|>0$. We call the 'ambiguity' of the choice of the monomials $z_{1}^{m-i p} z_{2}^{n+i q}$ the moving triangle ambiguity.

More generally, a moving triangle of a Newton diagram $\Gamma$ is a triangular face with vertices $P:=(p, 0,1), Q:=(0, q, 1)$, and $R:=(m, n, 0)$, where the edge $P Q$ is in some other face as well. Consider the line through $R$ parallel to $P Q$. Then (the moving vertex) $R$ can be replaced by any of the lattice points $S$ on this line with non-negative coordinates (or any collection of them). If $\Gamma$ satisfies $(2.5)$, then $\operatorname{gcd}(p, q)=1$, and by (A.2), $\overrightarrow{\mathbf{a}} \triangle=\left(a_{1}, a_{2}, a_{3}\right)=(q, p, m q+n p-p q)$. Therefore, one has

$$
\begin{aligned}
& p\left|m \Longleftrightarrow a_{2}\right| a_{3} \Longleftrightarrow R \text { can be replaced by a point on the } z_{2} \text { axis, } \\
& q\left|n \Longleftrightarrow a_{1}\right| a_{3} \Longleftrightarrow R \text { can be replaced by a point on the } z_{1} \text { axis. }
\end{aligned}
$$

\subsection{Stability of the invariants under the deformations}

Proposition 3.2.1. Consider two isolated singularities with non-degenerate Newton principal parts whose Newton boundaries are equivalent in the sense of Definition 3.1.3. Then the following invariants associated with these germs are the same:

(a) the Milnor number $\mu$;

(b) the link $K$;

(c) more generally, the embedded topological type;

(d) the spectral numbers (in particular, the geometric genus); the equivariant Hodge numbers;

(e) the multiplicity.

Moreover, a deformation associated with $M 1_{+}$or $M 2_{+}$admits a weak simultaneous resolution.

Proof. First of all, item (a) can be easily verified by direct computation (left to the reader) by Kouchnirenko's formula (2.4). Item (b) can also be checked directly from Oka's algorithm [Oka87] ( $\$ 4.2$ here), and item (e) is also elementary. However, there are also (more) conceptual short-cuts: the existence of a weak simultaneous resolution follows from a result of Oka [Oka89] (after we add some high degree monomials in the non-convenient case, and we note that our moves are 'negligible truncations' in the sense of Oka), which implies item (b) by a result of Laufer [Lau83]. For item (d) one can use Varchenko's result [Var82], which says that the spectrum is constant under a $\mu$-constant deformation. Note also that the geometric genus is the number of spectral numbers in the interval $(0,1]$. Finally, a $\mu$-constant $(f+t g)$-type deformation (cf. $\S 3.1 .6)$ is topological trivial by a result of Parusiński [Par99] (proving item (c)), and is equimultiple, for example, by Trotman [Tro80].

Remark 3.2.2. By a similar proof as in [Ste85] (valid for the spectrum), one can show that the set of spectral pairs (equivalently, the equivariant Hodge numbers) of $f$ are also determined by $\Gamma$, and are stable with respect to the $\sim$-deformation. Cf. also [Dan79].

Corollary 3.2.3. Fix a Newton diagram $\Gamma$ which satisfies (2.1). Then the following facts are equivalent: 


\section{INVARIANTS OF NEWTON NON-DEGENERATE SURFACE SINGULARITIES}

(a) $\{(0,1,1),(1,0,1),(1,1,0)\} \cap \Gamma \neq \emptyset$;

(b) $\Gamma$ is equivalent to a diagram which has no two-dimensional faces;

(c) $\Gamma$ is equivalent to the segment-diagram $[(0,1,1),(n, 0,0)]$ for some $n \geqslant 2$;

(d) $f_{\Gamma}(z):=\sum_{p \in \Gamma} a_{p} z^{p}$ (with generic coefficients $\left\{a_{p}\right\}_{p}$ ) is an $A_{n-1}$ singularity (the unique hypersurface cyclic quotient singularity with $\mu=n-1$ ) for some $n \geqslant 2$;

(e) the minimal dual resolution graph of $\left\{f_{\Gamma}=0\right\}$ is a string (with determinant $n$ ).

In fact, the integers $n$ in facts (c), (d), and (e) are equal.

Proof. (b) $\Rightarrow$ (c) follows from Examples 2.1.4 and 3.1.5. The implication (c) $\Rightarrow$ (b) is clear. For (a) $\Rightarrow(\mathrm{c})$, using Example 3.1.5, it is enough to prove that if $(0,1,1) \in \Gamma$, then $\Gamma \sim[(0,1,1)$, $(n, 0,0)]$ for some $n$. If $\Gamma$ intersects the $z_{3}$ axis at some point $(n, 0,0)$, then $[(0,1,1),(n, 0,0)] \subset \Gamma$ and one may use Example 3.1.4. Otherwise, one considers, as in Remark 2.1.5, the projection $\left(z_{1}, z_{2}, z_{3}\right) \mapsto\left(z_{1}, z_{2}\right)$ restricted to $\Gamma$. By $(2.1)$ there is at least one edge whose projection has the form $[(a, 0),(0,1)]$ (up to a permutation). Consider the edge-projection of this type which is closest to $(1,1)$, let its preimage in $\Gamma$ be $[(a, 0, c),(0,1, b)]$. This choice guarantees that $\Gamma$ contains the triangular face $\triangle$ with vertices $(a, 0, c),(0,1, b),(1,1,0)$. As $\triangle$, as a diagram, satisfies $(2.1), \Gamma \sim \triangle$ by Example 3.1.4. By M2 one can add to $\triangle$ the vertex $(a b+c, 0,0)$, and apply again Example 3.1.4 to show that $\triangle \sim[(0,1,1),(n, 0,0)]$ with $n=a b+c$.

Next, note that $a_{1} z_{1}^{n}+a_{2} z_{2} z_{3}\left(a_{1}, a_{2} \neq 0\right)$ defines an $A_{n-1}$ singularity. Hence, (c) $\Rightarrow$ (d) follows from Proposition 3.2.1(b), because the $A_{n-1}$ singularity is characterized by the fact that its link is the lens space $L(n, n-1)$. For $(\mathrm{d}) \Rightarrow(\mathrm{a})$, one uses that the quadratic part of the Taylor expansion of an $A_{n-1}$ singularity $f_{\Gamma}$ (in any coordinate system) has rank at least two.

(d) $\Leftrightarrow$ (e) follows from the fact that the $A_{n-1}$ singularities are the only hypersurface singularities whose minimal resolution graphs are strings.

3.2.4 Not all discrete analytic invariants of the germs remain constant under the above equivalence relation. The following example was provided by J. F. de Borbadilla, A. Melle-Hernández, and I. Luengo (private communication), in which Teissier's invariant $\mu^{*}$ jumps.

Example 3.2.5. Consider the deformation $f_{t}=z_{3}^{3}+z_{2}^{4} z_{1}+z_{1}^{10}+t z_{2}^{3} z_{3}$, which corresponds to Move 1, hence $f_{1}$ and $f_{0}$ are equivalent in the sense of Definition 3.1.3. However, the Milnor numbers of the generic hyperplane sections are not the same: $\mu^{(2)}\left(f_{1}\right)=7$, while $\mu^{(2)}\left(f_{0}\right)=8$. In particular, by [Lau87], this deformation does not admit a strong simultaneous resolution. A similar example was constructed by Briançon and Speder [BS75] (cf. also [Oka89]); the main difference is that in the present case the stable link $K\left(f_{1}\right)=K\left(f_{0}\right)$ is a rational homology sphere. Note also that $f_{0}$ is weighted homogeneous and $\operatorname{deg}\left(z_{2}^{3} z_{3}\right)>\operatorname{deg}\left(f_{0}\right)$. (This example also shows that [Ném04, Question 13.12] has a negative answer, i.e. for a deformation which admits a weak simultaneous resolution the existence of a strong simultaneous resolution is not guaranteed, even if the stable link is a rational homology sphere.)

Example 3.2.6. The recent manuscript [BLDM07, §4] provides a $\mu$-constant deformation of singularities with non-degenerate Newton principal part and $b_{1}(K)>0$ such that the homeomorphism of the tangent cone jumps, providing a counterexample to [Zar71, Conjecture B]. In fact, a counterexample also exists among rational homology spheres, e.g. the deformation $f_{t}=z_{1}^{3} z_{2}+z_{3}^{5}+z_{2}^{11}+t z_{1}^{2} z_{3}^{2}$ of type Move 1 . Then the homeomorphism type of $\left\{z_{1}^{3} z_{2}+t z_{1}^{2} z_{3}^{2}=0\right\}$ is not constant.

\subsection{Distinguished representatives}

3.3.1 In this section we assume that all of our Newton diagrams satisfy (2.1) and (2.5). It is preferable to have in each $\sim$-equivalence class a well-characterized and easily recognizable 


\section{G. Braun AND A. NÉmethi}

representative to work with. In its choice, we are guided by the following principles (motivated by Proposition 4.2.5, which states that such a 'minimal' diagram reflects better the minimal resolution graph $G_{\min }(f)$ of the germ $\left.f\right)$ :

(a) the representative should have a minimal number of faces;

(b) all of the faces which cannot be eliminated by M1_ should be 'minimized as much as possible' by $\mathrm{M} 2_{-}$;

(c) a representative may only contain a trapezoid if the trapezoid cannot be replaced by a triangle in its class.

This motivates the following.

Definition 3.3.2. A diagram $\Gamma$ is called M1-minimal if by the direct application of a move of type M1_ one cannot eliminate any of its faces.

Note that at least one M1-minimal representative exists in any equivalence class.

Also, one can decide on the M1-minimality of a diagram by analyzing the lattice points sitting on it, without any information about the other diagrams in its class. However, exactly for this reason, the above definition does not exclude the possibility that an M1-minimal diagram may have another diagram in its class with less faces. In fact, this may occur as the following example shows.

Example 3.3.3 (cf. the proof of Corollary 3.2.3). The diagram consisting of the unique triangular face with vertices $(a, 0, c),(0,1, b),(1,1,0)$ is M1-minimal, but it is equivalent to the segment $[(1,1,0),(0,0, a b+c)]$.

The next lemma guarantees that this is the only pathological case when such a phenomenon may occur. In the following $\#(\Gamma)$ denotes the number of faces of $\Gamma$.

Lemma 3.3.4. Fix a diagram $\Gamma$, which is not of type characterized by Corollary 3.2.3.

(a) Then $\Gamma$ is M1-minimal if and only if for any $\Gamma^{\prime} \sim \Gamma$ one has $\#\left(\Gamma^{\prime}\right) \geqslant \#(\Gamma)$.

(b) If $\Gamma$ and $\Gamma^{\prime}$ are both M1-minimal and $\Gamma \sim \Gamma^{\prime}$, then they can be connected by a sequence of diagrams related to each other only by moves $M 2_{ \pm}$. In particular, the set of supporting planes of the faces of the two diagrams are the same.

Note that the assumption is also essential for part (b); see, e.g., the segments of Example 3.1.5.

Proof. A sequence of diagrams $\Gamma_{1}, \Gamma_{2}, \ldots, \Gamma_{k}$ connects $\Gamma_{1}$ and $\Gamma_{k}$ if $\Gamma_{i}$ and $\Gamma_{i+1}$ (for $1 \leqslant i \leqslant k-1$ ) are related by one of the moves $\mathrm{M} 1_{ \pm}$or $\mathrm{M} 2_{ \pm}$, denoted by $\Gamma_{i} \stackrel{\mathrm{M} j_{ \pm}}{\longrightarrow} \Gamma_{i+1}$. Our goal is to replace a given sequence of diagrams connecting $\Gamma$ and $\Gamma^{\prime}$ by another sequence which has the additional property that all M1 - moves appear first. For this, we first analyze how one can modify two consecutive moves in a sequence, where the second is $\mathrm{M} 1_{-}$.

Fact. $\Gamma_{i} \stackrel{\mathrm{M} j_{ \pm}}{\longrightarrow} \Gamma_{i+1} \stackrel{\mathrm{M} 1_{-}}{\longrightarrow} \Gamma_{i+2}$ can be replaced either by moves $\Gamma_{i} \stackrel{\mathrm{M} 1_{-}}{\longrightarrow} \Gamma_{i+1}^{\prime} \stackrel{\mathrm{M} j_{ \pm}}{\longrightarrow} \Gamma_{i+2}$, or by a single move of type $\Gamma_{i} \stackrel{\mathrm{M} 1_{-}}{\longrightarrow} \Gamma_{i+2}$, or both moves can be eliminated, i.e. $\Gamma_{i}=\Gamma_{i+2}$.

Indeed, if the two moves operate on different faces of the diagram, then they can be performed in the reverse order with the same effect. So we can assume that the two moves operate on the same face $\triangle$.

If, additionally, the two moves have the same axis $A B$ (see Definition 3.1.3), then the moves eliminate one triangle from both sides of $A B$, hence $\Gamma_{i} \sim A B$ contradicting our assumption. Hence, the two axes are different. This can occur only if $\triangle$ is a triangle and the composition of the two moves is the removal of $\triangle$, i.e. a move of type M1_. This finishes the proof of the fact. 


\section{INVARIANTS OF NEWTON NON-DEGENERATE SURFACE SINGULARITIES}

The easy consequence of the fact is that if $\Gamma \sim \Gamma^{\prime}$, then they can be connected by a sequence $\Gamma_{i}$, in which all of the $\mathrm{M} 1$ - moves appear first (preceding the moves of other types). In particular, if $\Gamma$ is M1-minimal, then this sequence does not contain any M1_ moves, so the number of faces $\#\left(\Gamma_{i}\right)$ is non-decreasing along the sequence. This proves the non-trivial part of Lemma 3.3.4(a). If $\Gamma^{\prime}$ is also M1-minimal, then (applying the above also for the reverse sequence) $\#\left(\Gamma_{i}\right)$ must be constant, i.e. the sequence does not contain any move of type $\mathrm{M}_{ \pm}$, finishing the proof of part (b).

Definition 3.3.5 (Canonical and minimal representatives). Fix the equivalence class of a diagram which does not satisfy Corollary 3.2.3, and consider all M1-minimal representatives. By Lemma 3.3.4(b) they are related to each other by moves $\mathrm{M} 2_{ \pm}$. Clearly, this set has a unique maximal element with respect to $\mathrm{M} 2_{ \pm}$(or, equivalently, with respect to the inclusion). This diagram will be called the canonical representative of the class. It can be easily recognized: it is M1-minimal, and all of its faces are as large as possible.

The canonical representative satisfies the principle (a) of $\S 3.3 .1$, but not principle (b). For principle (b), we would need the unique minimal element with respect to $\mathrm{M} 2_{ \pm}$of all M1-minimal representatives; but such an element, in general, does not exist. Nevertheless, we consider the set of minimal elements (diagrams which cannot be reduced by $\mathrm{M} 2_{-}$) of all M1-minimal representatives. We call these representatives minimal. By Lemma 3.3.4, these are those representatives which cannot be reduced by any move $\mathrm{M} j_{-}$.

Example 3.3.6.

(a) Fix a trapezoidal face $\triangle$ of $\Gamma$ with vertices as in Lemma 2.3.2. One can remove the vertex $D$ if and only if either $n=1$ or $r_{2}+t q=1$. The vertex $A$ can be removed if and only if $r_{2}+q=1$. (There are analogous characterizations for $B$ and $C$, too.) The case $n=1$ is the 'moving triangle' situation Example 3.1.7. If $r_{2}+q=1$, then there are (at least) two possibilities for the choice of the axis of $\mathrm{M} 2{ }_{-}$, namely the segments $\left[(0,1, n),\left(r_{1}+t p, 0,0\right)\right]$ and $\left[(p, 0, n),\left(r_{1}+t p-p, 1,0\right)\right]$. One of them replaces the trapezoid by a triangle, while the other replaces it by a smaller trapezoid. Hence, in any situation, if a trapezoid can be decreased in some way, then it can be replaced by a triangle in the equivalence class of the diagram. Otherwise, it is called non-removable (this happens if $n>1, r_{1}+p>1, r_{2}+q>1$ ).

(b) If above $q=p=1$ and $r_{1}=r_{2}=0$, then $\triangle$ is the canonical representative of its class. One has four possible axes, and $\triangle$ can be reduced to the trapezoid $(0,1, n),(1,0, n),(t-1,1,0)$, $(1, t-1,0)$ or to the triangles $(0,1, n),(t, 0,0),(1, t-1,0)$ or $(1,0, n),(t-1,1,0),(0, t, 0)$. These are the minimal representatives.

Remark 3.3.7.

(a) Let $\triangle$ be a triangular face of an M1-minimal representative $\Gamma$. Then in any minimal representative of $\Gamma$, which is obtained from $\Gamma$ via moves $M 2_{-}, \triangle$ survives as a triangular face which is independent of the choice of the minimal representative. This happens because the axes of all the moves $\mathrm{M} 2_{-}$, which can be applied to $\triangle$, cannot intersect each other, hence all of them can be applied 'simultaneously' (a fact which is not true in the case of removable trapezoids, see Example 3.3.6).

(b) Therefore, any class whose canonical representative has a non-removable trapezoid, or a central triangle or a central edge, admits a unique minimal representative.

Discussion/Definition 3.3.8 (d-minimal representatives). Fix a class. It may contain many minimal representatives; we will distinguish one of them, and we call it d-minimal (distinguishedminimal). If the class admits a unique minimal representative, then there is no ambiguity for the choice. This happens, for example, in all of the situations of Remark 3.3.7(b). 


\section{G. Braun AND A. NÉmethi}

For the sake of completeness, we allow diagrams satisfying Corollary 3.2.3. For such a class, the (d-)minimal representative is the segment $[(0,1,1),(n, 0,0)]$, for some $n \geqslant 2$, as given in Corollary $3.2 .3(\mathrm{c})$.

Next, assume that a canonical representative contains a removable trapezoid (i.e. one replaceable by a triangle). Using the notation of Lemma 2.3.2, if $n>1$, then again there is a unique minimal representative, unless we are in the situation of Example 3.3.6(b) (when there are two, but they correspond to each other by a permutation of coordinates). By definition, this is the d-minimal representative (in the last case it is well-defined up to the permutation of coordinates).

If $n=1$, then we are in the situation of a moving triangle (Example 3.1.7), and the class may contain many minimal representatives. (An even more annoying fact is that such a class may contain two equivalent diagrams such that one of them has a central triangle while the other has a central edge.) We declare the position of the moving point $R$ for the d-minimal representative as follows. Assume that $p<q$ (for $q<p$ interchange $z_{1}$ and $z_{2}$ ). If $R$ cannot be moved to any of the coordinate axes (cf. (3.1)), then take for $R$ that possible lattice point which is closest to the $z_{1}$ axis. If $R$ can be moved to exactly one coordinate axis, then move it there. If $R$ can be moved to both axes, then move to the $z_{1}$ axis. (As the determinants of $Q R$ and $P R$ are $p$ and $q$, respectively, by this choice of $R$, the determinant of the edge lying on the coordinate plane is larger. There is no deep motivation for this choice, except that we need one. In the 'inverse' algorithm, the very same choice is built in.)

Corollary 3.3.9 (Structure of d-minimal representatives; cf. also Proposition 2.3.9). The d-minimal representative of an equivalence class (which does not satisfy Corollary 3.2.3) sits in exactly one of the following three disjoint families of diagrams characterized by the existence of:

(1) a non-removable trapezoid;

(2) a central triangle; or

(3) a central edge.

Notation 3.3.10. The three disjoint families listed in Corollary 3.3.9 will be denoted by $\mathbf{\square}, \mathbf{\Delta}, \mathbf{1}$. They can be divided further according to the number of hands. This number will appear as a subscript. For example, $\boldsymbol{}_{3}$ denotes that family of classes of Newton boundaries whose d-minimal representative has a non-removable trapezoid and three hands.

The first fruit of the minimality of a graph is the following arithmetical criterion.

Proposition 3.3.11. Fix a minimal representative $\Gamma_{\min }$ of a class which does not satisfy Corollary 3.2.3. Consider an edge of $\partial \Gamma_{\text {min }}$ which is the intersection of the faces $\triangle$ and $\nabla$ of $\Gamma_{\text {min, }+}$, where the second is non-compact. Then $n_{\triangle, \nabla}>1$.

Proof. We have to analyze two types of edge, cf. Remark 2.1.5. First we discuss edges on a coordinate plane, say $\left[\left(q_{1}, 0, q_{3}\right),\left(q_{1}^{\prime}, 0, q_{3}^{\prime}\right)\right]$. Take a triangle in $\triangle$ which satisfies the criterions of Lemma A.1.2(c). Then $n_{\triangle, \nabla}=n_{\triangle, \overrightarrow{\mathbf{e}_{\mathbf{2}}}}=p_{2}$. However, if $p_{2}=1$, then this triangle can be eliminated by $\mathrm{M} j_{-}$.

Now we turn to the other type of edges, which have the form $A B=[(a, 0, c),(0,1, b)]$ with $a>0$. Take a third vertex $C=(r, s, u)$ on $\triangle$ such that $\triangle_{A B C}$ is empty. Then the identity (A.8) of Lemma A.1.3 can be applied: $n_{\triangle, \nabla}=r+(s-1) a$. Assume that $r+(s-1) a=1$. If $s=0$, then $[(r, 0, u),(0,1, b)]$ is an axis of a move $\mathrm{M} j_{-}$, hence $\triangle_{A B C}$ can be eliminated. If $s=1$, then $r=1$, hence by $(2.5) u=0$, which contradicts our assumption about Corollary 3.2.3. The remaining case $s \geqslant 2$ imposes $r=0, s=2, a=1$, with $[(1,0, c),(0,2, u)]$ an axis of $\mathrm{M} j_{-}$which eliminates $\triangle_{A B C}$. 


\section{INVARIANTS OF NEWTON NON-DEGENERATE SURFACE SINGULARITIES}

Remark 3.3.12.

(a) By the above proof, when we eliminate triangles from a diagram by moves $\mathrm{M} j_{-}$, then, in fact, we eliminate those 'mixed determinants' (i.e. when a face is non-compact) with $n_{\triangle, \nabla}=$ 1. By Proposition 3.3.11, by repeated application of $\mathrm{M} j_{-}$, we can eliminate all such mixed determinants, provided that the class does not satisfy Corollary 3.2.3. (Otherwise this is not true: Example 3.3.3 shows a minimal triangle with a 'mixed determinant' 1.)

(b) The statement of Proposition 3.3.11 is also true for a class which satisfies Corollary 3.2.3 (where $\triangle$ and $\nabla$ are non-compact and contain $[(0,1,1),(n, 0,0)],): n_{\triangle, \nabla}=n>1$.

\section{The dual resolution graph}

\subsection{Graph terminology}

4.1.1 Recall that any resolution graph $G(f)$ of $\{f=0\}$ is also a possible plumbing graph of the link $K(f)$ of $f$. The link $K(f)$ is a rational homology sphere if and only if $G(f)$ is a tree, and the genera of all of the vertices are 0 . In such a case, $G(f)$ has only one set of decorations: each vertex carries the self-intersection number of the corresponding irreducible exceptional divisor. In this section we recall the terminology of resolution graphs, and we present a construction which 'simplifies' a given graph. Its output will be called the orbifold diagram.

4.1.2 Let $G$ be a decorated tree with vertices $\mathcal{V}$ and decorations $\left\{b_{v}\right\}_{v \in \mathcal{V}}$. The entries of intersection matrix $\left(I_{v w}\right)_{v, w \in \mathcal{V}}$ of $G$ are $I_{v v}=b_{v}$, and for $v \neq w$, one sets $I_{v w}=1$ if $[v w]$ is an edge and $I_{v w}=0$ otherwise. We assume that $I$ is negative definite (because the matrix of a dual resolution graph is so $[\mathrm{Mum} 61])$. By definition, $\operatorname{det}(G):=\operatorname{det}(-I)$ is the determinant of the graph $G$.

A node of $G$ is a vertex whose degree is at least 3 . Let $\mathcal{N}$ be their collection. A chain is the path between two nodes excluding the endpoints, which does not contain any nodes. (We say that the chain connects the two nodes.) Similarly, a leg of $G$ is a path between a degree 1 vertex and a node containing the degree 1 vertex but not the node, and containing no other nodes, either.

If $r, s \in \mathcal{N}$ are connected by a chain in $G$, then the determinant of this chain (i.e. the determinant of the corresponding subgraph) will be denoted by $n_{r s}$.

A star-shaped graph is a graph with a unique node. For any $r \in \mathcal{N}$, there is a unique maximal star-shaped subgraph $G_{r}$ of $G$ which contains $r$.

In general, a star-shaped graph is a plumbing graph of a Seifert 3-manifold. This has a natural $S^{1}$-action and orbifold structure. If the star-shaped graph $G_{r}$ has normalized Seifert invariants, say, $\left(\alpha_{i}, \omega_{i}\right)_{i}$ (here, each pair is associated with one of the legs of the subgraph, $\alpha_{i}$ is the leg-determinant, $0 \leqslant \omega_{i}<\alpha_{i}$, and we put the pair $(1,0)$ for legs with determinant one), and central vertex with decoration $b_{r}$, then the orbifold Euler number of $G_{r}$ is $e_{r}:=b_{r}+\sum_{i} \omega_{i} / \alpha_{i}$; see, e.g., [Wag83] for details.

4.1.3 The orbifold diagram. Sometimes we do not need all of the data of $G$, but only its shape and the determinants of some of its subgraphs. This information will be codified in a simpler graphlike diagram, the orbifold diagram associated with $G$, denoted by $G^{o}$.

The diagram $G^{o}$ is constructed from $G$ as follows: $G^{o}$ has vertices, edges connecting two vertices, and half-free edges. A half-free edge is attached with one of its ends to a vertex, while its other end is free. The vertices of $G^{o}$ are the nodes of $G$. The (ordinary) edges of $G^{o}$ are the chains of $G$. The endpoints of an edge are the two nodes it connects as a chain. The half-free edges are the legs of $G$. The endpoint of a half-free edge is the node to which it is adjacent in $G$ as a leg. Then we decorate $G^{o}$ : we put on each edge the determinant of the corresponding chain or leg, and we label 


\section{G. Braun AND A. NÉmethi}

each node $r$ with the orbifold Euler number $e_{r}$ of the star-shaped subgraph $G_{r}$. (In the special case when $G$ has no nodes, $G^{o}$ is a 'free' edge decorated by $\operatorname{det}(G)$.)

The half-free edges of the orbifold diagram $G^{o}$ will still be called legs.

The entries of the orbifold intersection matrix $\left(I_{r s}^{o}\right)_{r, s \in \mathcal{N}}$ of $G^{o}$, by definition, are $I_{r r}^{o}=e_{r}$, and for $r \neq s$ one sets $I_{r s}^{o}=1 / n_{r s}$ if $[r s]$ is an edge of $G^{o}$ and $I_{r s}^{o}=0$ otherwise. (Here we will not explain the 'orbifold geometry' behind this definition. Nevertheless, for a possible motivation, see $§$ 4.4.) Similarly as above, we set $\operatorname{det}\left(G^{o}\right):=\operatorname{det}\left(-I^{o}\right)$.

Lemma 4.1.4. Fix a graph $G$ as above with $\mathcal{N} \neq \emptyset$. Let $\Pi$ be the product of the determinants of all of the chains and legs of $G$. Then $I^{o}$ is negative definite, and

$$
\operatorname{det}(G)=\operatorname{det}\left(G^{o}\right) \cdot \Pi \text {. }
$$

Proof. The negative definiteness of $I^{o}$ follows from (4.1) applied to some subgraphs. The equality (4.1) is elementary linear algebra, it follows, for example, by induction on $\# \mathcal{N}$. If $\# \mathcal{N}=1$, then (4.1) is well-known, see, e.g., [Neu83]. The induction runs as follows. Fix $r, s \in \mathcal{N}$ which are connected by a chain $G_{r s}$. The connected components of $G \backslash\left(\{r, s\} \cup G_{r s}\right)$ are $\left\{G_{i}\right\}_{i}$, the connected component of $G \backslash\{r\}$ which contains $s$ is $G_{(s)}$, and similarly one defines $G_{(r)}$. Then $\operatorname{det}(G) \cdot \operatorname{det}\left(G_{r s}\right)=\operatorname{det}\left(G_{(s)}\right) \cdot \operatorname{det}\left(G_{(r)}\right)-\prod_{i} \operatorname{det}\left(G_{i}\right)$.

Remark 4.1.5. The orbifold diagram has exactly the same shape as the splice diagram considered in [NW02], but it has different decorations. Nevertheless, by similar identities as used in the proof of Lemma 4.1.4, one can show that the orbifold diagram contains the same amount of information as the splice diagram and $\operatorname{det}(G)$ altogether.

\subsection{Oka's algorithm for $G(f)$ and the case of minimal representatives}

4.2.1 Let $f:\left(\mathbb{C}^{3}, 0\right) \rightarrow(\mathbb{C}, 0)$ be a germ with isolated singularity and non-degenerate Newton principal part whose link $K(f)$ is a rational homology sphere. In particular, its Newton boundary $\Gamma(f)$ satisfies $(2.1)$ and $(2.5)$. In the first part of this section we recall the combinatorial algorithm of Oka [Oka87, Theorem 6.1], which provides a (possible, in general non-minimal) dual resolution graph $G(f)$ of the surface singularity $(\{f=0\}, 0)$ from $\Gamma(f)$.

In order to emphasize the dependence of the output upon $\Gamma(f)$, we write $G(\Gamma(f))$.

Notation 4.2.2. Recall that $\Gamma(f)$ is the union of compact faces of $\Gamma_{+}:=\Gamma_{+}(\operatorname{supp}(f))$, which can be recovered from $\Gamma(f)$ as $\Gamma_{+}(\{$vertices of $\Gamma(f)\})$. Hence, they contain the same amount of information. Similarly as above, $\mathcal{F}$ denotes the collection of all faces of $\Gamma_{+}$, and $\mathcal{F}_{c}$ denotes the set of all compact faces of $\Gamma_{+}$. For any $\triangle \in \mathcal{F}_{c}$, we write $\mathcal{F}_{\triangle}$ for the collection of all faces of $\Gamma_{+}$adjacent to $\triangle$. Other notation is from Notation/Definition 2.1.7.

4.2.3 The algorithm. The graph $G(\Gamma(f))$ is a subgraph of a larger graph $\widetilde{G}(\Gamma(f))$, whose construction is the following. To start with, we consider $\mathcal{F}$ as a set of vertices (we will call them face vertices). Then, if $\triangle, \nabla \in \mathcal{F}$ are two adjacent faces, then we connect them by $t_{\triangle, \nabla}$ copies of the following chain.

If $n_{\triangle, \nabla}>1$, then let $0<c_{\triangle, \nabla}<n_{\triangle, \nabla}$ be the unique integer for which

$$
\overrightarrow{\mathbf{c}}_{\triangle, \nabla}:=\left(\overrightarrow{\mathbf{a}}_{\nabla}+c_{\triangle, \nabla} \overrightarrow{\mathbf{a}}_{\triangle}\right) / n_{\triangle, \nabla}
$$

is an integral vector. Let us write $n_{\triangle, \nabla} / c_{\triangle, \nabla}$ as a continued fraction:

$$
\frac{n_{\triangle, \nabla}}{c_{\triangle, \nabla}}=b_{1}-\frac{1}{b_{2}-\frac{1}{\cdots-\frac{1}{b_{k}}}}
$$


INVARIANTS OF NEWTON NON-DEGENERATE SURFACE SINGULARITIES

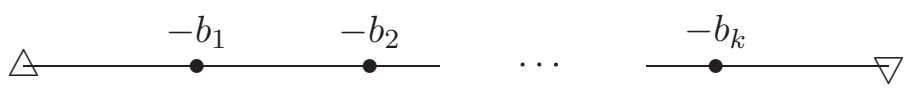

Figure 2. Chain between two face vertices.

where each $b_{i} \geqslant 2$. Then the chain with the corresponding self-intersection numbers is shown in Figure 2.

The left ends of all of the $t_{\triangle, \nabla}$ copies of the chain (marked by $\triangle$ ) are identified with the face vertex corresponding to $\triangle$, and similarly for the right ends marked by $\nabla$.

If $n_{\triangle, \nabla}=1$, then the chain consists of an edge connecting the vertices $\triangle$ and $\nabla$ (we put $t_{\triangle, \nabla}$ of them). Also, in this case we set $c_{\triangle, \nabla}:=0$ and $\overrightarrow{\mathbf{c}}_{\triangle, \nabla}:=\overrightarrow{\mathbf{a}}_{\nabla}$.

Next, we compute the decoration $b_{\triangle}$ of any face vertex $\triangle \in \mathcal{F}_{c}$ by the equation:

$$
b_{\triangle} \overrightarrow{\mathbf{a}}_{\triangle}+\sum_{\nabla \in \mathcal{F}_{\triangle}} t_{\triangle, \nabla} \overrightarrow{\mathbf{c}}_{\triangle, \nabla}=\overrightarrow{\mathbf{0}}
$$

What we get in this way is the graph $\widetilde{G}(\Gamma(f))$. Note that the face vertices corresponding to noncompact faces are not decorated. If we delete all of these vertices (and all of the edges adjacent to them) we get the dual resolution graph $G(\Gamma(f))$.

Note that $\widetilde{G}(\Gamma(f))$ has no legs, but some of the chains of $\widetilde{G}(\Gamma(f))$ become legs of $G(\Gamma(f))$ when we delete the vertices corresponding to $\mathcal{F} \backslash \mathcal{F}_{c}$. Regardless of whether a chain in Figure 2 transforms into a chain or a leg of $G(\Gamma(f))$, it keeps its determinant $n_{\triangle, \nabla}$.

Remark 4.2.4. If one starts with another Newton diagram, say $\Gamma^{\prime}(f)$, obtained from $\Gamma(f)$ via Moves 1 or 2, then the graph $G\left(\Gamma^{\prime}(f)\right)$ can be obtained from $G(\Gamma(f))$ by some blow-ups, in accordance with Proposition 3.2.1(b). Hence, in general, $G(\Gamma(f))$ is not a good minimal resolution graph. Recall that a dual resolution graph $G(f)$ with all genera vanishing is good minimal if all of its $(-1)$-vertices are nodes. Each normal surface singularity admits a unique good minimal resolution.

Proposition 4.2.5. If the Newton diagram $\Gamma_{\min }(f)$ is a minimal representative of its class, then the output $G\left(\Gamma_{\min }(f)\right)$ of Oka's algorithm is the good minimal resolution graph. In fact, $G\left(\Gamma_{\min }(f)\right)$ reflects the shape of the diagram $\Gamma_{\min }(f)$ (preserving the corresponding adjacency relations):

(a) the nodes of $G\left(\Gamma_{\min }(f)\right)$ correspond bijectively to the faces of $\Gamma_{\min }(f)$;

(b) the chains and legs of $G\left(\Gamma_{\min }(f)\right)$ correspond bijectively to the edges of $\Gamma_{\min }(f)$ not lying in $\partial \Gamma_{\min }(f)$ and the edges lying on $\partial \Gamma_{\min }(f)$, respectively (in the case of Corollary 3.2 .3 we understand by this that $\Gamma=\partial \Gamma$ is a segment, and $G\left(\Gamma_{\min }(f)\right)$ is a string).

Proof. The chains in Figure 2 contain no $(-1)$-vertex, any face has at least three edges, and all of the leg-determinants are greater than 1 by Proposition 3.3.11 and Remark 3.3.12, hence the statement follows.

The legs corresponding to different primitive segments of the same edge form a leg group.

In the next section we make a more direct connection between the normal vectors of faces, the coordinates of vertices of $\Gamma_{\min }(f)$, and the determinants of legs in $G\left(\Gamma_{\min }(f)\right)$.

\subsection{Leg-determinants in $G\left(\Gamma_{\min }(f)\right)$}

We fix a minimal Newton diagram $\Gamma_{\min }=\Gamma_{\min }(f)$ which does not satisfy Corollary 3.2.3, and let $\triangle \in \mathcal{F}_{c}$ be one of its faces. Let us consider the legs in $G\left(\Gamma_{\min }\right)$ adjacent to $\triangle$. By Proposition 4.2.5 they correspond to the primitive segments lying on the edges of $\partial \Gamma_{\min } \cap \triangle$. The following proposition 


\section{G. Braun AND A. NÉmethi}

summarizes the divisibility properties of the determinants of these legs. We refer to such a legdeterminant as the determinant $D(\alpha)$ of the corresponding edge $\alpha$. (The coordinate choices are accidental, they can be permuted arbitrarily.) As usual, we write $\overrightarrow{\mathbf{a}} \triangle=\left(a_{1}, a_{2}, a_{3}\right)$.

Proposition 4.3.1. We have the following.

(i) Any edge $\alpha$ of $\partial \Gamma_{\min } \cap \triangle$ satisfies the following divisibility properties.

(1) If $\alpha \subset\left\{z_{3}=0\right\}$, then $D(\alpha)=\operatorname{gcd}\left(a_{1}, a_{2}\right)$ and $\operatorname{gcd}\left(D(\alpha), a_{3}\right)=1$.

(2) If $\alpha=[(p, 0, a),(0,1, b)]$ crosses the $z_{3}$ axis, then $D(\alpha)=a_{3}$, and $D(\alpha)$ does not divide any of $\left\{a_{1}, a_{2}\right\}$, unless the edge can be 'moved' to a coordinate plane; see Example 3.1.7. If this happens, then $D(\alpha)$ divides the corresponding two coordinates as in part (i)(1), and either of the following cases holds.

(a) The edge belongs to a 'moving triangle' so that it can be moved to a coordinate plane by moving the moving vertex to a coordinate axis; see (3.1).

(b) The vertex $(0,2, c)$ is on $\triangle$, hence $\triangle$ can be extended (in the class of $\Gamma_{\text {min }}$ ) with a new vertex $(0,0,2 b-c)$, which lengthens the edge $\beta=[(0,1, b),(0,2, c)]$ by an extra primitive segment. We interpret this as moving $\alpha$ to this extra segment whose determinant is $D(\beta)$.

(ii) The determinants belonging to different edges of $\triangle$ satisfy the following.

(1) They differ, except in the case of part (i) (2)(b), where $D(\alpha)=D(\beta)$.

(2) They are pairwise relative prime except for the following two cases.

(a) An edge lying on a coordinate plane is adjacent to a crossing edge: the determinant of the former divides the determinant of the latter.

(b) An edge $\alpha$ lying on a coordinate plane is adjacent to two crossing edges: then $D(\alpha)$ is the greatest common divisor of the determinants of the crossing edges.

Proof. Part (i)(1) is clear by (2.2), because $\overrightarrow{\mathbf{a}} \triangle$ is primitive. Equation (A.7) implies the first part of item (i)(2). As $\overrightarrow{\mathbf{a}} \triangle$ is orthogonal to $\alpha$, i.e. $\left\langle\overrightarrow{\mathbf{a}}_{\triangle},(p, 0, a)\right\rangle=\left\langle\overrightarrow{\mathbf{a}}_{\triangle},(0,1, b)\right\rangle$, one has

$$
a_{2}=p a_{1}+(a-b) a_{3} .
$$

As $\overrightarrow{\mathbf{a}} \triangle$ is primitive $a_{3} \nmid a_{1}$ and if $a_{3} \mid a_{2}$, then $D(\alpha)=a_{3} \mid p$, too. This will be used later.

Let $P$ be a lattice point of $\triangle$, such that the triangle $\nabla$ (a part of the face $\triangle$ ) formed by $\alpha$ and $P$ is empty. First note that $P$ cannot be on the $z_{1} z_{3}$ plane, because then $\nabla$ would be removable. If $P$ lies on the $z_{2} z_{3}$ plane and has the form $(0, q, c)$, then, by (A.8), $D(\alpha)=(q-1) p$. If $D(\alpha) \mid p$, then $q=2$. This is the case (i)(2)(b).

Assume now that $P$ lies on the $z_{1} z_{2}$ plane, $P=\left(r_{1}, r_{2}, 0\right)$ with $r_{1}$ and $r_{2}$ positive. Then, again by (A.8), $D(\alpha)=r_{1}+p\left(r_{2}-1\right)$. Thus, if $D(\alpha) \mid p$, then $r_{2}=1$ and $r_{1} \mid p$. However, then the triangle $\nabla$ is movable (as in Example 3.1.7), and the vertex $(p, 0, a)$ can be moved to the point $\left(0,0, a+b p / r_{1}\right)$ lying on the third coordinate axis. This is the case (i) (2)(a).

For part (ii), assume that the leg-determinants belonging to two different edges are not relative prime. If one of the edges lies on a coordinate plane, then (2.2) and (A.7) show that we are in the situation of part (ii)(2)(a) or (ii)(2)(b). Otherwise, if one of the edges crosses, say, the $z_{1}$ axis, and the other edge crosses the $z_{2}$ axis, then their endpoints sitting on the $z_{1} z_{2}$ plane do not coincide, and hence case (ii)(2)(b) holds. Indeed, assume that the two endpoints do coincide. This common point cannot be $(1,1,0)$ by our assumption, cf. Corollary 3.2.3. Otherwise, by a relation similar to (4.5), the third coordinate of $\overrightarrow{\mathbf{a}} \triangle$ is an integral linear combination of the first two coordinates (which are the determinants), contradicting the fact that $\overrightarrow{\mathbf{a}} \triangle$ is primitive.

Finally, if two determinants are equal, then by part (ii)(2) the corresponding edges must be as in part (ii)(2)(a). However, then, by (A.6) and (A.8), we have $q=2$ which leads to part (i)(2)(b). 


\section{INVARIANTS OF NEWTON NON-DEGENERATE SURFACE SINGULARITIES}

COROLlary 4.3.2 (Non-removable trapezoids). The leg groups of a non-removable trapezoid have different determinants. Hence, the collection of chains and legs adjacent to the vertex corresponding to the trapezoid can be separated into four distinguishable groups.

As a vertex corresponding to a triangular face has at most three such groups of distinguishable legs and chains, the vertex of a non-removable trapezoid can be recognized in the resolution graph.

Proof of Corollary 4.3.2. Assume the contrary. Then by Proposition 4.3.1, we are in the situation of part (i) (2)(b) with the points $(p, 0, a),(0,1, b)$, and $(0,2, c)$ on the trapezoid (modulo a permutation of the coordinates). Then this face can be extended by the vertex $(0,0,2 b-c)$. This extended face is also a trapezoid. However, by Example 3.3.6(a), this is a removable trapezoid.

\subsection{The orbifold diagram}

4.4.1 We fix a minimal representative $\Gamma_{\min }(f)$ as in Proposition 4.2.5. Section 4.2 provides a good minimal resolution graph $G\left(\Gamma_{\min }(f)\right)$ from the Newton diagram $\Gamma_{\min }(f)$. On the other hand, to any graph $G$, the general procedure (see $\S 4.1 .3$ ) associates a diagram $G^{o}$. In the present situation this will be denoted by $G^{o}\left(\Gamma_{\min }(f)\right)$. Although, by the very construction of $G^{o}$, we (apparently) throw away some information, we prefer to use $G^{o}\left(\Gamma_{\min }(f)\right)$ because it reflects more faithfully the Newton diagram. For the convenience of the reader, we briefly sketch how one can draw $G^{o}\left(\Gamma_{\min }(f)\right)$ directly from $\Gamma_{\min }(f)$.

Similarly as in $\S 4.2$, first we construct a decorated graph $\widetilde{G}^{o}$. Its vertices are the elements of $\mathcal{F}$, i.e. all of the faces of $\Gamma_{\min ,+}$. If $\triangle, \nabla \in \mathcal{F}$ are adjacent in $\Gamma_{\min ,+}$, then we connect them by $t_{\triangle, \nabla}$ edges in $\widetilde{G}^{o}$, and we label each of these edges with the number $n_{\triangle, \nabla}$. Finally, we label each $\triangle \in \mathcal{F}_{c}$ with the orbifold Euler number of the maximal star-shaped subgraph containing $\triangle$, which is

$$
e_{\triangle}:=b_{\triangle}+\sum_{\nabla \in \mathcal{F}_{\triangle}} t_{\triangle, \nabla} \frac{c_{\triangle, \nabla}}{n_{\triangle, \nabla}} .
$$

In this way we get the labelled graph $\widetilde{G}^{o}$. If we remove the vertices $\left\{v_{\triangle}: \triangle \in \mathcal{F} \backslash \mathcal{F}_{c}\right\}$ (but we keep the edges, i.e. the new legs, adjacent to them), we get the diagram $G^{o}\left(\Gamma_{\min }(f)\right)$. For any $\triangle \in \mathcal{F}_{c}$, we call $e_{\triangle}$ the orbifold Euler number of $\triangle$.

4.4.2 The point is that (4.4) can be transformed via the orbifold Euler numbers into some (more natural) identities which only involve the normal vectors of the faces.

Proposition 4.4.3. Fix the representative $\Gamma_{\min }(f)$. Then for any $\triangle \in \mathcal{F}_{c}$, one has

$$
e_{\triangle} \overrightarrow{\mathbf{a}}_{\triangle}+\sum_{\nabla \in \mathcal{F}_{\triangle}} \frac{t_{\triangle, \nabla}}{n_{\triangle, \nabla}} \overrightarrow{\mathbf{a}}_{\nabla}=\overrightarrow{\mathbf{0}} .
$$

Proof. Use (4.2), (4.4), and (4.6).

Obviously, if one wishes to recover the equation of a face of $\Gamma_{\min }(f)$, one needs its normal vector $\overrightarrow{\mathbf{a}} \triangle$, and its face value, i.e. the value of $\overrightarrow{\mathbf{a}} \triangle$ on any of the face's point:

$$
m_{\triangle}:=\left\langle\overrightarrow{\mathbf{a}}_{\triangle}, P\right\rangle, \quad P \in \triangle \text {. }
$$

It turns out that these numbers $\left\{m_{\triangle}\right\}_{\triangle \in \mathcal{F}}$ also satisfy a similar equation.

Proposition 4.4.4. Fix $\Gamma_{\min }(f)$ as above. Then, for any $\triangle \in \mathcal{F}_{c}$, one has

$$
e_{\triangle} m_{\triangle}+\sum_{\nabla \in \mathcal{F}_{\triangle}} \frac{t_{\triangle, \nabla}}{n_{\triangle, \nabla}} m_{\nabla}=-g(\triangle) .
$$




\section{G. Braun AND A. NÉmethi}

Proof. Denote the vertices of $\triangle$ by $P_{0}, \ldots, P_{k}$ in this order, and set $P_{k+1}:=P_{0}$. Assume that $P_{i} P_{i+1}$ is the common edge of $\triangle$ and $\nabla$. Then, by (A.4), one has

$$
g\left(\triangle_{P_{0} P_{i} P_{i+1}}\right)=\frac{t_{\triangle, \nabla}}{n_{\triangle, \nabla}}\left\langle\overrightarrow{\mathbf{a}}_{\nabla}, P_{0}-P_{i}\right\rangle=\frac{t_{\triangle, \nabla}}{n_{\triangle, \nabla}}\left\langle\overrightarrow{\mathbf{a}}_{\nabla}, P_{0}\right\rangle-\frac{t_{\triangle, \nabla}}{n_{\triangle, \nabla}} m_{\nabla},
$$

with $g\left(\triangle_{P_{0} P_{0} P_{1}}\right)=g\left(\triangle_{P_{0} P_{k} P_{k+1}}\right)=0$. Then, by $(4.7)$ and $(4.10)$

$$
-e_{\triangle} m_{\triangle}=-e_{\triangle}\left\langle\overrightarrow{\mathbf{a}} \triangle, P_{0}\right\rangle=\sum \frac{t_{\triangle, \nabla}}{n_{\triangle, \nabla}}\left\langle\overrightarrow{\mathbf{a}}_{\nabla}, P_{0}\right\rangle=\sum \frac{t_{\triangle, \nabla}}{n_{\triangle, \nabla}} m_{\nabla}+\sum g\left(\triangle_{P_{0} P_{i} P_{i+1}}\right) .
$$

Then use the additivity of the combinatorial area.

Corollary 4.4.5. For any subset $\overline{\mathcal{F}}_{c} \subset \mathcal{F}_{c}$ consider the system of equations (4.7) for all $\triangle \in \overline{\mathcal{F}}_{c}$ in unknowns $\left\{\overrightarrow{\mathbf{a}}_{\triangle}: \triangle \in \overline{\mathcal{F}}_{c}\right\}$ (or in one of their fixed coordinates). Then this system is non-degenerate. The same is true for (4.9) instead of (4.7).

Proof. The matrix of the system (for $\overline{\mathcal{F}}_{c}=\mathcal{F}_{c}$ ) coincides with the matrix $I^{o}$ of the orbifold diagram $G^{o}\left(\Gamma_{\min }(f)\right)($ cf. $\S 4.1 .3)$, which is negative definite by Lemma 4.1.4.

Remark 4.4.6. If one wishes to solve the above equations, one needs the values for non-compact faces. If such a face is supported by a coordinate plane, then its normal vector is a coordinate vector, and its face value is 0 . Otherwise, if it has an edge of type $[(a, 0, c),(0,1, b)](a>0)$, then its normal vector is $(1, a, 0)$ and its face value is $a$.

The following lemma connects the face value of a central triangle with entries of normal vectors.

Lemma 4.4.7. Let $\triangle$ be an empty central triangular face with three adjacent faces $\nabla_{i}$. Write $n_{i}:=n_{\triangle, \nabla i}, \overrightarrow{\mathbf{a}}_{\nabla i}=\left(a_{1}^{(i)}, a_{2}^{(i)}, a_{3}^{(i)}\right)(1 \leqslant i \leqslant 3) ;$ and $\overrightarrow{\mathbf{a}}_{\triangle}=\left(a_{1}, a_{2}, a_{3}\right)$. Then

$$
-m_{\triangle}=a_{1} a_{2} a_{3} \cdot\left(e_{\triangle}+\sum_{i=1}^{3} \frac{a_{i}^{(i)}}{n_{i} a_{i}}\right) .
$$

Proof. Let $\left(0, p_{2}, p_{3}\right)$ be a vertex of $\triangle$. Then $m_{\triangle}=p_{2} a_{2}+p_{3} a_{3}$. By (A.3) (and by a sign check) $a_{1}^{(2)} a_{2}-a_{2}^{(2)} a_{1}=n_{2} p_{3}$ and $a_{1}^{(3)} a_{3}-a_{3}^{(3)} a_{1}=n_{3} p_{2}$. Use these identities and (4.7).

\section{Remark 4.4.8.}

(a) Of course, all of the results proved for $G\left(\Gamma_{\min }(f)\right)$ can be transformed into properties of $G^{o}=$ $G^{o}\left(\Gamma_{\min }(f)\right)$. For example, Proposition 4.2.5 reads as follows. The diagram $G^{o}$ reflects the shape and adjacency relations of $\Gamma_{\min }(f)$ : the vertices of $G^{o}$ correspond to the faces of $\Gamma_{\min }(f)$. The edges of $G^{o}$ connecting vertices correspond to the edges of $\Gamma_{\min }(f)$ not lying in $\partial \Gamma_{\min }(f)$, and the legs of $G^{o}$ correspond to the primitive segments lying on the edges of $\partial \Gamma_{\min }(f)$. By Proposition 3.3.11, all of the leg-decorations are greater than 1 , and they satisfy the divisibility properties of $\S 4.3 .1$.

Moreover, by the very definition, the intersection orbifold matrix $I^{o}$ (cf. $\S 4.1 .3$ ) can be read from $G^{o}$ and also the combinatorial areas $\{g(\triangle)\}_{\triangle \in \mathcal{F}_{c}}$, needed in (4.9). Indeed, $g(\triangle)+2$ equals the degree of the corresponding vertex in $G^{o}$ (cf. (2.3) and (2.5)).

(b) One may ask: how easily can $\Gamma_{\min }(f)$ be recognized from $G^{\circ}$ ? Well, rather difficulty! Already the types (1)-(3) of Corollary 3.3.9 are hardly recognizable. The 'easy' cases include (cf. Notation 3.3.10) recognized via Corollary 4.3.2, or $\boldsymbol{\Lambda}_{3}\left(G^{o}\right.$ has a vertex adjacent with three other vertices). In these cases, one also recognizes the vertices corresponding to hands (vertices adjacent with one vertex), or to central faces. Also, if $G^{o}$ has one vertex, then it corresponds to a one-faced diagram. However, all of the other families cannot be easily separated. For example, it is hard to separate the case of a central triangle with two non-degenerate arms 


\section{INVARIANTS OF NEWTON NON-DEGENERATE SURFACE SINGULARITIES}

from the case of a central edge. In these cases, it is not easy at all to find the hands or central triangles.

Another difficulty arises as follows. Consider an arm (with many triangles) crossing, say, the $z_{3}$ axis. There are two types of triangles in it, depending on whether the non-crossing edge is on the $z_{1} z_{3}$ or $z_{2} z_{3}$ plane. These types are invisible from the shape of $G^{o}$ (and will be determined using technical arithmetical properties of the decorations).

\subsection{The main result}

\section{Starting the inverse algorithm}

5.1.1 We consider analytic germs $f:\left(\mathbb{C}^{3}, 0\right) \rightarrow(\mathbb{C}, 0)$ with isolated singularity at 0 , with nondegenerate Newton principal part, and with rational homology sphere link $K(f)$. At a combinatorial level, this means that we consider all of the Newton boundaries $\Gamma$ with (2.1) and (2.5). Oka's algorithm $\S 4.2$ provides a resolution graph $G(\Gamma)$ for each such $\Gamma$. Such a graph, in general, is not good minimal. However, Proposition 4.2.5 guarantees that if some graph $G$ can be obtained by this procedure, then also the good minimal resolution graph $G_{\min }$ associated with $G$ (obtained from $G$ by repeated blow-downs of $(-1)$-vertices of degree less than 3 ) can be obtained by running the algorithm for a minimal representative $\Gamma_{\min }$ of $\Gamma$.

Recall that all of the resolution graphs which are equivalent modulo blowing up/down (-1)vertices can be regarded as the plumbing graphs of the same plumbed 3-manifold, the link $K(f)$. By [Neu81], this class of graphs, and the unique good minimal graph, can be recovered from the oriented topological type of the link $K(f)$.

Recall also that to any graph $G$ one can associate the orbifold diagram $G^{o}$.

Our next result, which also implies Theorem 1.0.1 from the introduction, says that Oka's algorithm is, basically, injective.

TheOrem 5.1.2. The $d$-minimal representative $\Gamma_{\min }$ (up to a permutation of coordinates) can be uniquely recovered from the orbifold diagram $G^{o}$ associated with $G\left(\Gamma_{\min }\right)$.

Corollary 5.1.3. We consider germs as in $§$ 5.1.1. Then one has the following.

(i) The orbifold diagram associated with the good minimal resolution contains the same information as $G_{\text {min }}$ itself.

(ii) If the links of two germs $f_{0}$ and $f_{1}$ are homeomorphic, then there exist germs $\left\{g_{i}\right\}_{i=0}^{k}$ (as in $\S 5.1 .1)$ and a coordinate-permutation $\sigma$ so that $g_{0}=f_{0}, g_{k}=f_{1} \circ \sigma$, and $g_{i}+t\left(g_{i+1}-g_{i}\right)$ $(0 \leqslant i<k)$ is a $\mu$-constant deformation corresponding to one of the moves $\mathrm{M} j_{ \pm}$.

5.1.4 Outline of the algorithm. The inverse algorithm which recovers the d-minimal representative from $G^{o}$ is rather long. It distinguishes three cases depending on the number $N$ of nodes.

(i) The case $N=0$ : the minimal resolution graph has no nodes. This is the simplest case solved in Corollary 3.2.3. Let $n$ denote the determinant (equivalently, $G^{o}$ is a 'free' edge with decoration $n$ ). The representative is the diagram of $z_{1}^{n}+z_{2} z_{3}$.

(ii) The case $N=1$ : the minimal resolution graph is star-shaped (equivalently, $G^{o}$ has only one vertex). This case corresponds to (deformations of) isolated weighted homogeneous germs. Then [MN05] proves that from the resolution graph one can recover the supporting plane $\pi$ of the unique face of the (representative) Newton boundary (or the weights). Next we provide an even shorter argument. By [Pin77], the Poincaré series of the graded algebra of the germ $f$ is recovered from $G(f)$. However, this is a rational function of type $\left(t^{m}-1\right) /\left(\left(t^{a_{1}}-1\right)\right.$ $\left.\left(t^{a_{2}}-1\right)\left(t^{a_{3}}-1\right)\right)$ codifying the equation $\sum a_{i} z_{i}=m$ of $\pi$, cf. [Wag83]. Putting all of the possible lattice points on $\pi$, we get the canonical representative of $\Gamma$. 


\section{G. Braun And A. NÉmethi}

A long combinatorial case-by-case verification also recovers $\pi$ from $G^{o}$, which the patient reader may rediscover using the classification in Appendix A.2.

(iii) The case $N>1$ : the orbifold diagram has at least two vertices. This is the subject of the remaining sections; an outline of it is given here. The procedure involves three main technical steps:

(1) arm preprocessing, which provides partial information about the arms and about the face(s) behind the shoulders $(\S 5.2)$;

(2) determination of the center ( $\S \S 5.4,6$ and 7 );

(3) arm postprocessing which calculates the arms completely ( $\$ 5.3)$.

In fact, step (1) for an arm runs only if we know the position of the corresponding hand, otherwise it should be preceded by a hand-search step.

In the next two sections we discuss the easier arm preprocessing and postprocessing, respectively. They are uniform no matter how the diagram looks.

On the other hand, we devote more sections to determine the center, because it distinguishes many cases depending on how the center looks and uses a separate algorithm in every case.

\subsection{Arm preprocessing}

Definition 5.2.1. Let us consider a non-degenerate arm of a d-minimal Newton diagram in the direction, say, of the $z_{3}$ axis. Its basic data consists of the following:

(1) the correspondence $\kappa$ between the triangles and edges of the arm and the corresponding vertices and decorated edge groups of $G^{o}$, respectively;

(2) the first and second coordinates of the vertices of the triangles of the arm;

(3) the third coordinates of the normal vectors of all of the triangles of the arm and also of the (compact or non-compact) face of $\Gamma_{+}$opposite the shoulder;

(4) the face values of the non-compact faces adjacent to the triangles of the arm associating these numbers to the corresponding 'half-free' edges of $G^{o}$.

The basic data is an invariant of the arm, i.e. it is independent of the parts of $\Gamma$ outside the arm, and also does not depend on the choice of coordinates; explicit coordinates are used in the definition only for simplicity of language. In particular, in the language of parts (1)-(4) above, it is only welldefined up to a permutation of the first two coordinates. Nevertheless, this permutation is global: if we exchange the coordinates in one triangle, then we have to exchange in all of them. (In part (4) the face values are independent of the permutation of the first two coordinates. In fact, they are 0 except for perhaps one leg of the hand.)

It is convenient to distribute the basic data among the triangles. The basic data of a triangle $\triangle$ of an arm (in the direction of the $z_{3}$ axis) consists of the first and second coordinates of its vertices and the third coordinate of its normal vector, and also the correspondence $\kappa$ between the edges of $\triangle$ in $\Gamma$ and edge groups of $\kappa(\triangle)$ in $G^{o}$. The basic data of a triangle is part of the basic data of the containing arm; the choice of coordinates agrees with the choice of coordinates for the arm. (If a triangle is contained in several arms, then it has a separate basic data for each of the containing arms.)

5.2.2 The aim of arm preprocessing. Assume that we identify in $G^{o}$ the vertex corresponding to the hand of an arm in the d-minimal representative. The aim of arm preprocessing is to determine from $G^{o}$ the basic data of this arm. We compute the basic data of the triangles of the arm one after the other beginning at the hand. Meanwhile, we will also recognize when we reach the shoulder of the arm, and we will compute the third coordinate of the normal vector of the next face as required by Definition 5.2.1(3). 


\section{INVARIANTS OF NEWTON NON-DEGENERATE SURFACE SINGULARITIES}

5.2.3 We start with the basic data of the hand $\triangle$. At this stage we are free to make any choice of coordinates: we assume that the arm is in the direction of $z_{3}$ axis; and if $\triangle$ has any edge with interior lattice points (say, $t-1$ of them), then this edge sits on the $z_{2} z_{3}$ plane. Let $\kappa(\Delta)$ be the corresponding vertex in $G^{o}$. The following section collects some facts about decorations of the legs adjacent to $\kappa(\Delta)$. By Proposition 3.3.11, all of them are greater than 1 .

5.2.4 If $\triangle$ intersects the $z_{3}$ axis, then there are two types of leg-decorations (cf. Proposition 4.2.5): $t$ legs decorated with $n_{\triangle, \overrightarrow{\mathbf{e}_{1}}}$ and one leg with $n_{\triangle, \overrightarrow{\mathbf{e}_{2}}}$. Note that by Proposition 4.3.1(ii) one has gcd $\left(n_{\triangle, \overrightarrow{\mathbf{e}_{1}}}, n_{\triangle, \overrightarrow{\mathbf{e}_{2}}}\right)=1$, and by (A.6) the vertices of $\triangle$ have the form $(0,0, *),\left(0, t n_{\triangle, \overrightarrow{\mathbf{e}_{2}}}, *\right)$, and $\left(n_{\triangle, \overrightarrow{\mathbf{e}_{1}}}, 0, *\right)$. This last fact together with (A.5) implies $a_{3}=n_{\triangle, \overrightarrow{\mathbf{e}_{1}}} \cdot n_{\triangle, \overrightarrow{\mathbf{e}_{2}}}$.

Otherwise, if $\triangle$ has an edge of type $[(a, 0, c),(0,1, b)](a>0)$, set $\overrightarrow{\mathbf{n}}:=(1, a, 0)$ as in Lemma A.1.3. Then there are $t$ legs with decoration $n_{\triangle, \overrightarrow{\mathbf{e}_{1}}}$ and one leg with $n_{\triangle, \overrightarrow{\mathbf{n}}}$. By (A.7) we have $n_{\triangle, \overrightarrow{\mathbf{n}}}=a_{3}$, by (A.6) we have $n_{\triangle, \overrightarrow{\mathbf{e}_{\mathbf{1}}}}=a$, and by (A.8) we have $n_{\triangle, \overrightarrow{\mathbf{e}_{\mathbf{1}}}} \mid n_{\triangle, \overrightarrow{\mathbf{n}}}$ (because $r=0$ ). Again, by (A.8), the vertices of $\triangle$ have the form $(0,1, *),\left(n_{\triangle, \overrightarrow{\mathbf{e}_{1}}}, 0, *\right)$ and $\left(0,1+t n_{\triangle, \overrightarrow{\mathbf{n}}} / n_{\triangle, \overrightarrow{\mathbf{e}_{1}}}, *\right)$. Note that $n_{\triangle, \overrightarrow{\mathbf{n}}}=n_{\triangle, \overrightarrow{\mathbf{e}_{1}}}$ may happen only in the case (i)(2)(b) of Proposition 4.3.1.

For the corresponding face values, see Remark 4.4.6.

5.2.5 Algorithm: the basic data of a hand. Let $N$ be the set of all of the decorations of the legs adjacent to $\kappa(\Delta)$. One may have the following situations.

(a) The situation $N=\left\{n_{1}, n_{2}\right\}$ with $\operatorname{gcd}\left(n_{1}, n_{2}\right)=1$. One of them, say $n_{2}$, decorates exactly one leg; the other, $n_{1}$, decorates several legs, say $t$ of them. (If $t=1$, then the construction is symmetric.) Then (up to a permutation of the coordinates, cf. Definition 5.2.1) the vertices of the hand have the form $(0,0, *),\left(0, t n_{2}, *\right)$, and $\left(n_{1}, 0, *\right)$; and the third coordinate of $\overrightarrow{\mathbf{a}}_{\triangle}$ is $n_{1} n_{2}$. All of the face values are 0 .

(b) The situation $N=\left\{n_{1}, n_{2}\right\}$ with $n_{1} \mid n_{2}$. Then the number of legs decorated by $n_{1}$ will be denoted by $t$, and (automatically) $n_{2}$ decorates one leg. The face value of legs with $n_{1}$-decoration is 0 , but the face value of the unique $n_{2}$-decorated leg is $n_{1}$. The vertices of the hand have the form $(0,1, *),\left(n_{1}, 0, *\right)$, and $\left(0,1+t n_{2} / n_{1}, *\right)$, and the third coordinate of $\overrightarrow{\mathbf{a}} \triangle$ is $n_{2}$.

(c) The situation $N=\{n\}$. Then let $t+1$ be the total number of legs. We set $n_{1}=n_{2}=n$. Then the basic data of the hand is given by the same formulas as in situation (b). We separate one leg (with face value $n$ ), the others form another group (with face value 0 ).

5.2.6 Arm continuation. Assume that we have computed from $G^{o}$ the basic data of the triangles $\triangle_{1}, \ldots, \triangle_{k}$ (belonging to an arm in the direction of $z_{3}$, where $\triangle_{1}$ is the hand, and $\triangle_{i}$ is adjacent to $\triangle_{i+1}$ ) in such a way that the coordinate-ambiguities are compatible (i.e. if we fixed coordinates for $\triangle_{1}$, then for all of the other $\triangle_{i}$ we respect the same choice). Our aim is to determine the part of the basic data corresponding to the next face, i.e. the third coordinate of its normal vector, and whether it belongs to the arm. If it does, then we also compute its basic data.

We write $\triangle:=\triangle_{k}$ and set $\kappa(\triangle)$ for the corresponding vertex of $G^{o}$. By the inductive step, we have already computed the correspondence $\kappa$ of all of the edges of $\triangle$ with the edges adjacent to $\kappa(\triangle)$. Let $\nabla$ be the next face of $\Gamma_{+}$, adjacent to $\triangle$, and set $\gamma:=\nabla \cap \triangle$. The face $\nabla$ is compact if and only if $\kappa(\gamma)$ connects two vertices of $G^{o}$, one of them is obviously $\kappa(\triangle)$. If this is the case, we set $\kappa(\nabla)$ for the other end.

In any situation, we need the third coordinate $a_{3}$ of the normal vector $\overrightarrow{\mathbf{a}}_{\nabla}$. This can be computed from $G^{o}$ and the basic data of the triangles $\triangle_{i}$ using (4.7).

Next, if $\nabla$ is compact, we wish to decide whether it belongs to the arm. The face $\nabla$ is a non-removable trapezoid if and only if $\kappa(\nabla)$ admits four distinguishable groups of adjacent edges 


\section{G. Braun And A. NÉmethi}

(cf. Corollary 4.3.2). In this case, clearly, $\nabla$ does not belong to the arm. The same is true if $\kappa(\nabla)$ has no legs (which happens if and only if $\nabla$ is a central triangle). Therefore, assume that $\nabla$ is a triangle with at least one adjacent leg.

Lemma 5.2.7. The face $\nabla$ belongs to the arm if and only if $\kappa(\nabla)$ has an adjacent leg whose decoration divides $a_{3}$.

Proof. Write $\gamma=A B$, and let $C$ be the third vertex of $\nabla$. If $C$ is on the $z_{2} z_{3}$ or $z_{1} z_{3}$ planes, then the leg associated with $B C$ or $A C$ divides $a_{3}$ by Proposition 4.3.1(i)(1). If $C$ is not sitting on one of these two planes, then it can only have a leg if at least one of $A C$ and $B C$ is a crossing edge. However, by Proposition 4.3.1(i)(2), such an edge determinant only divides $a_{3}$ if $\nabla$ is a moving triangle whose moving vertex $C$ can be moved to a coordinate axis. However, this would contradict the definition of the d-minimal representatives in Discussion/Definition 3.3.8.

If $\nabla$ does not belong to the arm, then we stop (having all of the basic data of the arm).

Next, assume that $\nabla$ belongs to the arm. Then we have to identify edges of $\nabla$ with edges adjacent to $\kappa(\nabla)$ in $G^{o}$, and to determine the first two coordinates of $C$.

First we identify the leg-decoration $n_{\nabla}$, adjacent to $\kappa(\nabla)$, which corresponds to the edge $\alpha$ of $\nabla$ which lies on a coordinate plane. By Proposition 4.3.1(i)(1), it divides $a_{3}$. We claim that $n_{\nabla}$ is the largest leg-decoration adjacent to $\kappa(\nabla)$ which divides $a_{3}$. Indeed, we only have to check the case when $\kappa(\nabla)$ has two leg-decorations $N=\left\{n_{1}, n_{2}\right\}$ (the determinants of $A C$ and $B C$, one edge sitting on a coordinate plane, the other being a crossing edge), both dividing $a_{3}$. Then, by Proposition 4.3.1, $\nabla$ is a moving triangle such that $C$ can be moved to both coordinate axes, and by the construction of the d-minimal representative (cf. Discussion/Definition 3.3.8), the determinant of the edge which lies on the coordinate plane is larger.

Now, we fix an edge of $\triangle$ (whose determinant will be denoted by $n_{\triangle}$ ) which lies on a coordinate plane (which is either $z_{1} z_{3}$ or $z_{2} z_{3}$, determined clearly by the basic data of $\triangle$ ). Denote this plane by $\pi$. Then, by Lemma A.1.4, $\alpha$ lies on $\pi$ if and only if $n_{\triangle}=n_{\nabla} \mid n_{\triangle, \nabla}$.

This is also valid for $C$, hence this clarifies whether $C$ is on the $z_{1} z_{3}$ or $z_{2} z_{3}$ plane. Finally, we have to compute the first two coordinates of $C$. One of them is 0 (depending whether $\pi$ is the $z_{1} z_{3}$ or $z_{2} z_{3}$ plane), the other can be determined using (A.5).

Then we add $\nabla$ to the triangles $\left\{\triangle_{i}\right\}_{i}$ and repeat the 'arm continuation' process by induction.

\subsection{Arm postprocessing}

5.3.1 The arm postprocessing step assumes knowledge of two sets of data: the first is the basic data coming from arm preprocessing, the second is some knowledge about the face on the other side of the shoulder (which is usually but not always, the center). More precisely, let $\nabla$ be the face in $\Gamma_{+}$containing the shoulder of the arm, but not contained in the arm. (It is non-compact if and only if the edge $\kappa(\nabla)$ in $G^{o}$ is a leg.)

(i) The first set of data: the basic data of an arm in the direction of the $z_{3}$ axis modulo the ambiguity of a permutation of the first two coordinates. Recall that the basic data of the arm also includes the knowledge of $\left\langle\overrightarrow{\mathbf{a}_{\nabla}}, \overrightarrow{\mathbf{e}_{\mathbf{3}}}\right\rangle$.

(ii) The second set of data: consists of all of the coordinates of $\overrightarrow{\mathbf{a}}_{\nabla}$ and of the shoulder of the arm in some choice of coordinates $z_{1}$ and $z_{2}$. The coordinate $z_{3}$ is the same as in the first set.

Hence, by assumption, we have a 'half-compatibility' connecting the two choices of coordinates in the two sets of data: the third coordinate $z_{3}$ in the basic data (i) and for the pair $\left(\overrightarrow{\mathbf{a}}_{\nabla}\right.$, shoulder) in set (ii) are matched. However, a priori, we do not know how to identify the other (i.e. the first two) coordinates in the two sets of data. 


\section{INVARIANTS OF NEWTON NON-DEGENERATE SURFACE SINGULARITIES}

The aim of arm postprocessing is (using sets (i) and (ii) and $G^{o}$ ) to compute all of the coordinates of the vertices of the arm in a unified choice of coordinates for the sets of data.

5.3.2 Unifying the first two coordinates. Let $z_{1}, z_{2}, z_{3}$ be the coordinates in which we describe the second set of data (ii). Assume that in these coordinates the endpoints of the shoulder are $A=\left(0, p_{2}, p_{3}\right)$ and $B=\left(q_{1}, 0, q_{3}\right)$. (Note that the data (i) recognizes the first two coordinates up to their permutation of $A$ and $B$. Hence, if $p_{2} \neq q_{1}$, then this information already unifies the coordinates. However, in general, we have to do more.)

Let $\triangle$ be the last triangle of the arm (i.e. $\triangle \cap \nabla=A B$ ). Fix an edge $\alpha$ of $\triangle$ in $\Gamma$ whose edge-group $\kappa(\alpha)$ in $G^{o}$ contains, say, $t$ legs. We will determine whether $\alpha$ is on the $z_{1} z_{3}$ or $z_{2} z_{3}$ plane: this will orient all of the basic data (i) in accordance with $\left\{z_{i}\right\}_{i}$.

First we compute (in the coordinates $\left\{z_{i}\right\}_{i}$ ) the normal vector $\overrightarrow{\mathbf{a}}_{\triangle}$. For this, note that $n_{\triangle, \nabla}$. $\overrightarrow{A B}=\overrightarrow{\mathbf{a}}_{\triangle} \times \overrightarrow{\mathbf{a}}_{\nabla}$. This follows from Lemma A.1.2(a) up to a sign; the sign is a consequence of the right-hand rule for vector products. As $\overrightarrow{A B}, \overrightarrow{\mathbf{a}}_{\nabla}$ and $n_{\triangle, \nabla}$ are known, this identifies $\overrightarrow{\mathbf{a}} \triangle$ up to a summand of a scalar multiple of $\overrightarrow{\mathbf{a}}_{\nabla}$. As $\left\langle\overrightarrow{\mathbf{a}_{\triangle}}, \overrightarrow{\mathbf{e}_{\mathbf{3}}}\right\rangle$ is also known (from data (i)) and $\left\langle\overrightarrow{\mathbf{a}}_{\nabla}, \overrightarrow{\mathbf{e}}_{\mathbf{3}}\right\rangle$ is not 0 , these facts determine $\overrightarrow{\mathbf{a}} \triangle$ completely.

Now, we determine whether $\alpha$ lies on $z_{1} z_{3}$ or $z_{2} z_{3}$ plane. Recall that from the basic data (i), we know the set of the first two coordinates of $\alpha$ : one of them is 0 , the other is, say, $\delta(\alpha)>0$. For example, if $\alpha=\left[\left(q_{1}, 0, q_{3}\right),\left(q_{1}^{\prime}, 0, q_{3}^{\prime}\right)\right]$, then $\delta(\alpha)=q_{1}-q_{1}^{\prime}$, and $\delta(\alpha) / t$ is a positive integer, known from the basic data (i). Then (A.5) and (2.2), for $i \in\{1,2\}$, reads as

$$
\alpha \subset z_{i} z_{3} \text { plane } \Rightarrow a_{3}=\operatorname{gcd}\left(a_{3}, a_{i}\right) \cdot \delta(\alpha) / t .
$$

As $\overrightarrow{\mathbf{a}} \triangle$ is primitive, and the above greatest common divisor is, in fact, a leg-determinant, hence it is greater than 1 by Proposition 3.3.11, the right-hand side of the above identity cannot be true for both $i=1$ and 2 simultaneously. This fact determines which coordinate plane contains $\alpha$.

5.3.3 The complete determination of the arm. Now, using $\S 5.3 .2$, we can write all of the basic data (i) in the coordinates $z_{1}, z_{2}, z_{3}$ of (ii). Note that the basic data (i) determines completely all of the normal vectors and all of the face values associated with the non-compact faces adjacent to the arm (cf. Remark 4.4.6). Moreover, from data (ii) we know the normal vector and the face value of $\nabla$. Hence, the affine equations of all the triangles in the arm follow from the systems in Corollary 4.4.5 (where $\overline{\mathcal{F}}_{c}$ is the index set of triangles of the arm).

\subsection{The complete inverse algorithm for $\Delta_{3}$}

We end this section with a clarification of the case $\boldsymbol{\Delta}_{3}$. First note that this family can be identified using the diagram $G^{o}$ : it has a (unique) vertex $v$ with three adjacent vertices and without any legs. In this section we assume that $G^{o}$ has this property.

The vertex $v$ corresponds to the central triangle $\triangle$. The other vertices can be grouped in three, each group consisting of a string of adjacent vertices corresponding to the three arms of the diagram. The hands correspond to vertices with exactly one adjacent vertex.

We mark the three vertices corresponding to the hands (or directions of the arms) with the three coordinates. Here we are free to make any marking (up to a permutation of the coordinates). We fix one. Once this choice is made, let us denote the coordinates of $\triangle$ by $\left(0, p_{2}, p_{3}\right),\left(q_{1}, 0, q_{3}\right)$, and $\left(r_{1}, r_{2}, 0\right)$. At this stage these entries are unknowns.

Now, we preprocess the arms. For example, for the arm in the direction of $z_{3}$ we obtain the basic data of that arm up to a permutation of the first two coordinates. In particular, we obtain: 


\section{G. Braun AND A. NÉmethi}

(a) the first two coordinates of the shoulder $\left[\left(0, p_{2}, p_{3}\right),\left(q_{1}, 0, q_{3}\right)\right]$ up to a permutation, hence the set $\mathcal{S}=\left\{q_{1}, p_{2}\right\}$

(b) $\left\langle\overrightarrow{\mathbf{a}} \triangle, \overrightarrow{\mathbf{e}_{3}}\right\rangle$;

(c) the face values of the legs of this arm.

Summing up for all three arms, we obtain:

(A) the pairs of coordinates $\left\{r_{2}, q_{3}\right\},\left\{q_{1}, p_{2}\right\},\left\{p_{3}, r_{1}\right\}$;

(B) $\overrightarrow{\mathbf{a}}_{\triangle}$;

(C) the face values of all of the legs and, hence, also the face value $m_{\triangle}$ of $\triangle$, from the system (4.9) via Corollary 4.4.5.

Lemma 5.4.1. Let $\left(0, p_{2}, p_{3}\right),\left(q_{1}, 0, q_{3}\right)$, and $\left(r_{1}, r_{2}, 0\right)$ be the vertices of an empty triangle $\triangle$ (with $\left.p_{2}, p_{3}, q_{1}, q_{3}, r_{1}, r_{2}>0\right)$. Then these coordinates are uniquely determined by:

(i) the sets $\left\{r_{2}, q_{3}\right\},\left\{q_{1}, p_{2}\right\},\left\{p_{3}, r_{1}\right\}$;

(ii) the normal vector $\left(a_{1}, a_{2}, a_{3}\right)=\overrightarrow{\mathbf{a}} \triangle$ of $\triangle$;

(iii) the face value $m_{\triangle}$ of $\triangle$.

Coming back to our original situation, Lemma 5.4.1 determines the central triangle $\triangle$. Then we postprocess the arms to calculate all of the missing data about $\Gamma$.

Proof of Lemma 5.4.1. We have a $\mathbb{Z}_{2}$-ambiguity for each set of part (i) and we wish to select the correct choice from the $2^{3}$ possibilities. For this, first assume that we are able to decide which element of the set $\left\{r_{2}, q_{3}\right\}$ is $r_{2}$ and which is $q_{3}$. Then we claim that the other two ambiguities disappear. Indeed, the face value identities written for the vertices of $\triangle$

$$
a_{1} q_{1}+a_{3} q_{3}=a_{2} p_{2}+a_{3} p_{3}=a_{1} r_{1}+a_{2} r_{2}=m_{\triangle}
$$

(where $a_{k}>0$ for all $k$ ) and part (i) provide $\triangle$. Hence, we have at most two choices: either the correct one for every arm or the wrong one for every arm (i.e. when we interchange $r_{2}$ with $q_{3}$ and $q_{1}$ with $p_{2}$ and $p_{3}$ with $r_{1}$ ). We claim that the wrong choice can be ruled out. Indeed, assume that both choices of system of integers satisfy the formula (A.2) for $\overrightarrow{\mathbf{a}} \triangle$ and (5.1). Note that, without loss of generality, we may assume that $r_{1}$ is the smallest among $r_{1}, p_{2}$, and $q_{3}$. Write (a part of) (A.2) and (5.1) for both choices:

$$
\begin{aligned}
p_{2} q_{3}+r_{2} p_{3}-r_{2} q_{3} & =q_{1} r_{2}+q_{3} r_{1}-q_{3} r_{2}=a_{1}, \\
q_{3} r_{1}+p_{3} q_{1}-p_{3} r_{1} & =r_{2} p_{3}+r_{1} p_{2}-r_{1} p_{3}=a_{2}, \\
a_{2} p_{2}+a_{3} p_{3}=a_{1} r_{1}+a_{2} r_{2} & =m_{\triangle}=a_{2} q_{1}+a_{3} r_{1}=a_{1} p_{3}+a_{2} q_{3} .
\end{aligned}
$$

Then (5.2) implies that $0 \leqslant\left(p_{2}-r_{1}\right) / r_{2}=\left(q_{1}-p_{3}\right) / q_{3}$, hence $q_{1} \geqslant p_{3}$ too. Thus,

$$
a_{2}=q_{3} r_{1}+p_{3} q_{1}-p_{3} r_{1} \geqslant r_{1}^{2}+p_{3}^{2}-r_{1} p_{3}=\left(r_{1}-p_{3}\right)^{2}+r_{1} p_{3}>\left|r_{1}-p_{3}\right| .
$$

On the other hand, from (5.4) expressing $a_{1}$ and $a_{3}$ yields $a_{1}=a_{2}\left(q_{3}-r_{2}\right) /\left(r_{1}-p_{3}\right)$ and $a_{3}=$ $a_{2}\left(p_{2}-q_{1}\right) /\left(r_{1}-p_{3}\right)$. However, this contradicts the fact that $\overrightarrow{\mathbf{a}} \triangle$ is primitive:

$$
\operatorname{gcd}\left(a_{1}, a_{2}, a_{3}\right)=\frac{a_{2}}{\left|r_{1}-p_{3}\right|} \operatorname{gcd}\left(q_{3}-r_{2}, r_{1}-p_{3}, p_{2}-q_{1}\right)>1 \text {. }
$$

\section{The inverse algorithm for the families}

\subsection{The start}

6.1.1 By Corollary 4.3.2, the family can be identified from $G^{o}$ : it has a (unique) vertex $v$ with four different types of edges. In this section we assume that $G^{o}$ has this property. 


\section{INVARIANTS OF NEWTON NON-DEGENERATE SURFACE SINGULARITIES}

The vertex $v$ corresponds to a non-removable trapezoid $\triangle$. This vertex always has at least one leg group (corresponding to the bottom edge).

Lemma 6.1.2. The diagram has at least one non-degenerate arm.

Proof. Assume that the top edge is the shoulder of a degenerate arm. Then, write the coordinates of the vertices $\triangle$ as in Lemma 2.3.2. Then (up to a permutation of the first two coordinates) $q=1$, and by Example 3.3.6, $r_{2}>0$ and $n>1$. For $r_{2}=1$, the trapezoid $\triangle$ can be enlarged and is removable. Hence, $\Gamma$ has a non-degenerate arm in the direction of $z_{1}$.

The hands can also be identified in $G^{o}$ : they are those vertices (different from $v$ ) which have one adjacent vertex. The next algorithm splits according to their number.

Note also, that by Lemma A.1.1, and with the notation of Lemma 2.3.2, the normal vector of $\triangle$ is

$$
\overrightarrow{\mathbf{a}}_{\triangle}=\left(n q, n p, r_{1} q+r_{2} p+(t-1) p q\right) .
$$

\subsection{The case of three non-degenerate arms: $\boldsymbol{D}_{3}$}

This case has many similarities with $\S 5.4$; but, in fact, it is simpler because the legs of $v$ help in the procedure. Let the decoration of the unique leg group of $v$ be $d$.

We start by preprocessing the three arms. This provides the coordinates of $\overrightarrow{\mathbf{a}} \triangle$ (up to a permutation). As we already identified the vertex of the central face, we know when we arrive at the shoulder. Nevertheless, at this step, we see a difference between the side arms and the top arm. Consider, for example, a side arm and the 'hidden' triangle (as part of $\triangle$ ) formed by the shoulder and the base edge. With this triangle the arm continuation procedure of $\$ 5.2 .6$ is not obstructed, in other words, (by Lemma 5.2.7) $d$ divides the corresponding coordinate of $\overrightarrow{\mathbf{a}} \triangle$. For the top arm this is not the case.

Therefore, $d$ divides exactly two coordinates of $\overrightarrow{\mathbf{a}} \triangle$. We attach the coordinate $z_{3}$ to the arm for which this divisibility does not hold (in this way its shoulder will be the top edge and the bottom edge will sit on the $z_{1} z_{2}$ plane). The coordinates $z_{1}$ and $z_{2}$ (chosen arbitrarily) will be attached to the other two strings of vertices.

As the face values of the legs of $v$ are 0 , and all of the other face values associated with legs have been determined during arm preprocessing, (4.9) and Corollary 4.4.5 provide the face value of $\triangle$. In particular, we get the equation of the affine plane supporting $\triangle$. As the top edge is primitive (and parallel to the $z_{1} z_{2}$ plane), this is enough for its identification. In particular, with the notation of Lemma 2.3.2, we get $n, p$, and $q$ (in fact, $n=d$ by (6.1)). During preprocessing of the arm in the direction $z_{1}$, we have obtained the set $\left\{n, r_{2}\right\}$, but $n$ is already identified, hence we also obtain $r_{2}$. Similarly, we get $r_{1}$. Thus, we know all of the vertices of $\triangle$, hence the algorithm finishes by postprocessing the arms.

\subsection{The case of two non-degenerate arms: $\boldsymbol{\square}_{\mathbf{2}}$}

6.3.1 We have two different leg groups (one of them attached to the bottom) and two nondegenerate arms. First we have to determine whether the shoulders of the non-degenerate arms are the side edges, or one of them is the top edge. This can be decided by the following divisibility property. Its proof uses (6.1) and the fact that $\overrightarrow{\mathbf{a}} \triangle$ is primitive (the details are left to the reader).

Lemma 6.3.2. Consider a trapezoid $\triangle$ with coordinates as in Lemma 2.3.2. Assume that it has two non-degenerate arms. Then (up to permutation of the first two coordinates) there are two possibilities. 


\section{G. Braun AND A. NÉmethi}

(i) Case 1: $q=1, n>1, r_{1}>1$ and $r_{2}>1$, the direction of the non-degenerate arms are the $z_{1}$ and $z_{2}$ axes, and $\overrightarrow{\mathbf{a}}_{\triangle}=\left(a_{1}, a_{2}, a_{3}\right)=(n, n p, *)$, hence $a_{1} \mid a_{2}$.

(ii) Case 2: $r_{1}=0$ or $r_{1}=1$, and $n>1, p>1, q>1$, the direction of the non-degenerate arms are the $z_{1}$ and the $z_{3}$ axes, but the coordinates $\left(a_{1}, a_{2}, a_{3}\right)$ of $\overrightarrow{\mathbf{a}} \triangle$ do not satisfy any divisibility relation: $a_{1} \nmid a_{3}, a_{3} \nmid a_{1}$.

Thus, the algorithm starts by preprocessing the two non-degenerate arms to get two coordinates of $\overrightarrow{\mathbf{a}} \triangle$. If one of them divides the other then we are in Case 1 above, otherwise we are in Case 2. Next we treat each case independently.

6.3.3 Case 1: side edges as shoulders of non-degenerate arms. Preprocessing the arms has provided (say) the first two coordinates of the normal vector $\overrightarrow{\mathbf{a}} \triangle$. We name the coordinate axes so that the smallest of the first two coordinates of $\overrightarrow{\mathbf{a}} \triangle$ is the first coordinate. Then we compute $n$ as the first coordinate and $p$ as the fraction of the first two coordinates. As $q=1$, at this point, we know all of the coordinates of the top edge. Then we end this case by the same argument as in $\S 6.2$. Preprocessing of the arm in the direction $z_{1}$ has provided the set $\left\{n, r_{2}\right\}$. As $n$ is already identified, we obtain $r_{2}$. Similarly, we also obtain $r_{1}$. Finally, $t+1$ is the number of legs of $\triangle$. Knowing all of the vertices of $\triangle$, we finish by postprocessing the arms.

6.3.4 Case 2: top edge as shoulder of a non-degenerate arm. Here, one may proceed in the spirit of the other cases of $\S \S 6.2$ and 6.3 .3 , but one may use the following observation as well. We may think about this situation as the degeneration of $\boldsymbol{\Delta}_{3}$ (cf. $\S 5.4$ ). Indeed, consider the trapezoid $\triangle$ as in Lemma 2.3.2 and cut it into two triangles along $\left[\left(r_{1}+t p, r_{2}, 0\right),(0, q, n)\right]$. Let $\triangle_{1}$ denote the lower triangle (whose vertices are $(0, q, n),\left(r_{1}+t p, r_{2}, 0\right)$, and $\left.\left(r_{1}, r_{2}+t q, 0\right)\right)$, and let $\triangle_{2}$ denote the upper triangle. We may consider $\triangle_{1}$ as a 'virtual' hand with two different leg groups, and $\triangle_{2}$ as a central triangle with two 'genuine' and one 'virtual' arms. The degeneration consists of the fact that $\triangle_{1}$ and $\triangle_{2}$ are in the same plane. Nevertheless, we can apply the same argument. The basic data of the 'virtual hand', similarly as in $\S 5.2 .5$, together with the basic data of the 'genuine' arms provide all of the data necessary to apply Lemma 5.4.1 for the empty triangle $\triangle_{2}$. Therefore, we obtain $\triangle_{2}$ (up to a permutation of the coordinates). Postprocessing the two arms and completing $\triangle_{2}$ to a trapezoid (in its supporting plane) ends the procedure.

\subsection{The case of one non-degenerate arm: $\square_{1}$}

The central vertex is attached to one non-degenerate arm and three leg groups. We denote the set of decorations of these legs by $\mathcal{D}$, which contains three different elements (cf. Corollary 4.3.2). Preprocessing of the arm provides a coordinate of $\overrightarrow{\mathbf{a}} \triangle$, denoted by $A$, and two coordinates of the shoulder (see, e.g., part (a) of $\S 5.4$ ) forming the set $\mathcal{S}$. The above discussions (and/or Appendix A.1) determine these three objects $A, \mathcal{D}, \mathcal{S}$ in terms of the integers used in Lemma 2.3.2 for the trapezoid $\triangle$. Basically (up to a permutation of the first two coordinates), there are two possibilities (depending on whether the shoulder of the arm is a side or top edge) as follows (for the coordinates $\left(a_{1}, a_{2}, a_{3}\right)$ of $\overrightarrow{\mathbf{a}} \triangle$ see (6.1), which shows that $\operatorname{gcd}\left(a_{3}, n\right)=1$; moreover, if $r_{1}=0$, then $p \mid a_{3}$, and if $r_{2}=0$, then $\left.q \mid a_{3}\right)$ :

(i) $q=1, r_{2}>1, p>1, n>1$, and $r_{1}=0$ or $r_{1}=1$, and the (minimal) diagram has an arm in the direction of the $z_{1}$ axis; then, $A=a_{1}=n, \mathcal{S}=\left\{n, r_{2}\right\}$, and $\mathcal{D}=\left\{n, a_{3}, n^{r_{1}} p\right\}$; if $r_{1}=0$, then $\operatorname{gcd}(n, p)=1$;

(ii) $p>1, q>1$, but $r_{1}$ and $r_{2}$ are 0 or 1 , and the arm is in the direction of the $z_{3}$ axis; then $A=a_{3}, \mathcal{S}=\{p, q\}$, and $\mathcal{D}=\left\{n, n^{r_{1}} p, n^{r_{2}} q\right\}$, where $\operatorname{gcd}(p, q)=1$. 


\section{INVARIANTS OF NEWTON NON-DEGENERATE SURFACE SINGULARITIES}

The first case satisfies $A \in \mathcal{S} \cap \mathcal{D}$. If $A \in \mathcal{S} \cap \mathcal{D}$ happens in case (ii), then $r_{1}=0$ and $r_{2}=t=1$ (or vice versa), and $\triangle$ is a parallelogram with two sides on coordinate planes. Hence, by a permutation of the $z_{1}$ and $z_{3}$ axes one arrives at the situation (i).

Analyzing the above data, one derives the following algorithm to recover $\triangle$ from $G^{o}$. It has two cases.

(1) If $A \in \mathcal{S} \cap \mathcal{D}$, then set $n=A$ and $r_{2}$ is the other element of $\mathcal{S}$. The arm is in the direction of $z_{1}$. Two subcases may occur:

(a) if there exists $D \in \mathcal{D} \backslash\{n\}$ with $n \mid D$, then set $r_{1}=1$ and $p=D / n$;

(b) if $\mathcal{D} \backslash\{n\}=\left\{D_{1}, D_{2}\right\}$ with $D_{1} \mid D_{2}$, then set $r_{1}=0$ and $p=D_{1}$.

(2) If $A \notin \mathcal{S} \cap \mathcal{D}$, then we obtain $n$ as the smallest element of the set $\mathcal{D} \backslash\{$ all divisors of $A$ \}. The set $\mathcal{S}$ has two (relative prime) elements; we declare them (arbitrarily) $p$ and $q$ (hence, we will get $\triangle$ up to a permutation of the first two coordinates). Then $r_{1}$ and $r_{2}$ are determined by the fact that $\mathcal{D}=\left\{n, n^{r_{1}} p, n^{r_{2}} q\right\}$. The arm is in the direction of the $z_{3}$ axis.

In this way, we recover $\triangle$ and the position of the arm in both cases, hence the algorithm ends by postprocessing the arm.

\section{The inverse algorithm for the remaining cases $\Delta_{1}, \Delta_{2}, 1_{1}, 1_{2}$}

\subsection{Find a hand!}

7.1.1 Next we assume that $G^{o}$ has at least two vertices, each vertex has at most two adjacent vertices, and for each vertex the number of adjacent vertices and leg groups together is three. An end vertex has one adjacent vertex. The diagram $G^{0}$ has two end vertices. The minimal subgraph generated by vertices and edges connecting them is a string.

Clearly, the Newton diagram has at least one hand. Compared with the previous cases, now it is much harder to recognize the vertices of $G^{o}$ corresponding to hands (and/or centers). A hand always corresponds to an end vertex, but end vertices may also correspond to central triangles (e.g. the case of a moving triangle), or to the last triangle (adjacent to the shoulder) of an arm (e.g. the diagram of $z_{3}^{a} z_{2}+z_{1}^{b}+z_{1}^{c} z_{2}^{d}+z_{2}^{e}$ ).

For any end vertex $v$, consider its two leg groups. Let $t_{i}$ and $n_{i}$ denote the number of legs and the decoration of the leg groups for $i=1,2$. Set $r(v):=n_{1} t_{2}+n_{2} t_{1}$.

By Proposition 4.3.1(ii)(2), if the two decorations of an end vertex $v$ are not relative prime, then $v$ is a hand. We call such an end vertex an easily recognizable hand (ER-hand).

Lemma 7.1.2. Assume that neither of the end vertices $v_{1}$ and $v_{2}$ of $G^{o}$ is an ER-hand. We mark one of them as follows. If $r\left(v_{1}\right)<r\left(v_{2}\right)$, then $v_{1}$ is marked. If $r\left(v_{1}\right)=r\left(v_{2}\right)$, then the one with greater orbifold Euler number is marked. If even their orbifold Euler numbers are equal, then $G^{o}$ has only two vertices (namely, $v_{1}$ and $v_{2}$ ) and it has an isomorphism permuting these two vertices. Then we mark arbitrarily one of the vertices.

All in all, the marked vertex is always a hand (in the last case up to this isomorphism).

Example 7.1.3. The symmetric case occurs if the Newton diagram has only four vertices $(0,0,2)$, $(p, 0,1),(0, q, 1)$, and $\left(r_{1}, r_{2}, 0\right)$ (satisfying $\left.r_{1} q+r_{2} p>2 p q\right)$. Then $G^{o}$ has two vertices, each having two legs decorated by $p$ and $q$. One can check that even the resolution graph is symmetric. This is surprising because the Newton diagram is not symmetric at all: either face is a hand, the other is a moving (central) triangle. Nevertheless, the algorithm recovers the asymmetric Newton diagram from a symmetric orbifold diagram! (Up to permutation of coordinates, this is the only possibility for the symmetric case, see the proof below.) 


\section{G. Braun AND A. NÉmethi}

Proof of Lemma 7.1.2. Fix a non-degenerate arm in the direction of the $z_{3}$ axis with hand $v_{1}$. Then the sum of the first two coordinates of the crossing edges of this arm strictly increases from the hand to the shoulder. For the first segment (closest to $v_{1}$ ) it is $r\left(v_{1}\right)$, cf. $\S 5.2 .4$. Assume that $v_{2}$ corresponds to the triangle $\triangle_{P Q R}$, with $P=\left(0, p_{2}, p_{3}\right), Q=\left(q_{1}, 0, q_{3}\right)$, and $R=\left(r_{1}, r_{2}, 0\right)$. We may assume that $p_{3}>0$ and $q_{3}>0$ (otherwise $v_{2}$ is a hand and we have nothing to prove). Thus, it is enough to show $q_{1} \leqslant \operatorname{det}(P Q)$ (and its analogue). As by (A.8), this determinant is $a_{2}$ (of $\triangle_{P Q R}$ ), and $a_{2}=q_{3} r_{1}+p_{3}\left(q_{1}-r_{1}\right)$ by (A.2), we need $q_{1} \leqslant q_{3} r_{1}+p_{3}\left(q_{1}-r_{1}\right)$. By $(2.1)$ at least one of $p_{3}$ and $r_{1}$ is 1 , hence the inequality follows. Moreover, $r\left(v_{1}\right)=r\left(v_{2}\right)$ if and only if $v_{1}$ and $v_{2}$ are the only vertices of $G^{o}$, and $p_{3}=q_{3}=1$; hence, the Newton diagram is given by $(0,0, c),\left(0, p_{2}, 1\right),\left(q_{1}, 0,1\right),\left(r_{1}, r_{2}, 0\right)$. For this, using $\S 4.4$, we get $e_{v_{1}} \geqslant e_{v_{2}}$, with equality if and only if $c=2$. For $c=2$ the graph is symmetric.

7.1.4 Start of the algorithm. We fix an end vertex $v_{1}$ which corresponds to a hand. We denote the other end vertex by $v_{2}$ (we may not know yet whether it is a hand). The algorithm starts with preprocessing the arm with hand $v_{1}$. Depending on the outcome, we continue by $\S 7.1 .5, \S 7.2$, or $\oint 7.3$.

7.1.5 The arm contains all vertices. We assume that the arm of $v_{1}$ contains all of the vertices of $G^{o}$. We fix the coordinates in such a way that the arm is in the direction of $z_{3}$. Then the shoulder has the form $\left[\left(r_{1}, 0,0\right),\left(0, p_{2}, p_{3}\right)\right]$ with $p_{3}=0$ or $p_{3}=1$, and $r_{1}>1$. Let $\overrightarrow{\mathbf{a}}$ be the normal vector (of the non-compact face) beyond the shoulder. With this choice, preprocessing the arm has provided the set $\left\{r_{1}, p_{2}\right\}$ and the third coordinate $a_{3}$ of $\overrightarrow{\mathbf{a}}$. Note that if $p_{3}=0$, then $\overrightarrow{\mathbf{a}}=\overrightarrow{\mathbf{e}_{\mathbf{3}}}$, otherwise $\overrightarrow{\mathbf{a}}=\left(1,0, r_{1}\right)$. Hence, if $a_{3}=1$, then $p_{3}=0$, but if $a_{3}>1$, then $p_{3}=1$ and $r_{1}=a_{3}$. In the $p_{3}=1$ case we get the integers $r_{1}$ and $p_{2}$, but in the case $p_{3}=0$, the integers $r_{1}$ and $p_{2}$ behave symmetrically, so we distinguish them arbitrarily. The algorithm finishes by postprocessing the arm.

Note that this algorithm covers not only the family $\mathbf{1}_{1}$, but also some part of $\mathbf{1}_{2}$. The remaining classes of $\mathbf{1}_{2}$ will be discussed in $\S 7.3 .2$ (in accordance with this paragraph).

\subsection{The case $\Delta_{1}$}

7.2.1 We assume that the arm of $v_{1}$ contains all vertices but one, which is not an ER-hand. Assume that the arm is in the direction $z_{3}$, and let $\left[\left(q_{1}, 0, q_{3}\right),\left(0, p_{2}, p_{3}\right)\right]$ be its shoulder with $p_{2} \geqslant 2$, $q_{1} \geqslant 2$. As $v_{2}$ is not an ER-vertex, $p_{3}>0$ and $q_{3}>0$ (cf. $\S 5.2 .4$ ). If the third vertex of the face associated with $v_{2}$ is $\left(r_{1}, r_{2}, 0\right)$, then $r_{i}>0(i=1,2)$ because otherwise $v_{2}$ would be in the arm of $v_{1}$. Therefore, $v_{2}$ corresponds to a central triangle with only crossing edges. Moreover, (2.1) guarantees that $1 \in\left\{r_{1}, p_{3}\right\} \cap\left\{r_{2}, q_{3}\right\}$. Let $\left(a_{1}, a_{2}, a_{3}\right)$ be the normal vector of the face of $v_{2}$.

Let us collect some facts about such a Newton diagram in order to be able to find the right algorithm. As $r_{1}=r_{2}=1$ is not possible (see $\S 5.1 .4(\mathrm{i})$ ), we may assume that $p_{3}=1$. (This introduces a choice of the coordinates $z_{1}$ and $z_{2}$, and at this moment it is not clear how this choice fits with any property of $G^{o}$; this will be explained later.)

We distinguish two cases. The first case is $q_{3}=1$, then $v_{2}$ is a moving triangle, hence $a_{1}=p_{2}$ and $a_{2}=q_{1}$. The second case is $q_{3}>1$, which we analyze in the rest of this paragraph. As $1 \in\left\{r_{2}, q_{3}\right\}$, we get $r_{2}=1$. By (A.2) one has

$$
\left(a_{1}, a_{2}, a_{3}\right)=\left(p_{2} q_{3}-q_{3}+1, q_{3} r_{1}+q_{1}-r_{1}, r_{1} p_{2}+q_{1}-q_{1} p_{2}\right) .
$$

From this and $q_{3} \geqslant 2$ one obtains $a_{1} \geqslant 2 p_{2}-1>p_{2}-1$ and $a_{2} \geqslant r_{1}+q_{1}>r_{1}, q_{1}$. In particular, $a_{1}+a_{2}>p_{2}+q_{1}$ and hence $\left\{a_{1}, a_{2}\right\} \neq\left\{p_{1}, q_{2}\right\}$. The face value computed via the two vertices $\left(r_{1}, 1,0\right)$ 
and $\left(0, p_{2}, 1\right)$ gives $r_{1} a_{1}+a_{2}=p_{2} a_{2}+a_{3}$. Therefore, the integers $r_{1}, p_{2}, q_{1}, a_{1}, a_{2}$ satisfy

$$
\begin{gathered}
a_{3}=r_{1} a_{1}-\left(p_{2}-1\right) a_{2}, \\
0<r_{1}<a_{2}, \\
0<p_{2}-1<a_{1} .
\end{gathered}
$$

7.2.2 The algorithm. The two decorations of the legs of $v_{2}$ are $\mathcal{D}=\left\{a_{1}, a_{2}\right\}$ (cf. (A.7)), where $\operatorname{gcd}\left(a_{1}, a_{2}\right)=1$ by Proposition 4.3.1(ii). Preprocessing the arm (with hand $v_{1}$ ) has produced $a_{3}$ and the set $\mathcal{S}=\left\{p_{2}, q_{1}\right\}$ (we cannot distinguish the two coordinates yet). We compute the coordinates of $v_{2}$ below, and then postprocess the arm to determine the rest of the Newton diagram.

We distinguish two cases for computing $v_{2}$. The first case is $\mathcal{D}=\mathcal{S}$. Let the two elements of this set be $a_{1}=p_{2}$ and $a_{2}=q_{1}$ (there is a choice here between the $z_{1}$ and $z_{2}$ coordinates). We select the d-minimal (as explained in Discussion/Definition 3.3.8) solution $\left(r_{1}, r_{2}\right)$ of positive integers of the equation $r_{1} p_{2}+r_{2} q_{1}-q_{1} p_{2}=a_{3}$. (The only reason for selecting the d-minimal solution is to obtain the d-minimal representative.) Then the vertices of $v_{2}$ are $\left(q_{1}, 0,1\right),\left(0, p_{2}, 1\right),\left(r_{1}, r_{2}, 0\right)$.

The second case is $\mathcal{D} \neq \mathcal{S}$. We choose the unique 6 -tuple $\left(r_{1}, p_{2}, q_{1}, q_{3}, a_{1}, a_{2}\right)$ of positive integers with $\mathcal{S}=\left\{a_{1}, a_{2}\right\}, \mathcal{D}=\left\{p_{2}, q_{1}\right\}$ satisfying both (7.1) and (7.2). (Uniqueness will be proved in the next section.) Then the vertices of $v_{2}$ (up to a permutation of the first two coordinates) are $\left(q_{1}, 0, q_{3}\right),\left(0, p_{2}, 1\right)$ and $\left(r_{1}, 1,0\right)$.

7.2.3 Uniqueness of the 6-tuple. Note that once the choice between $a_{1}$ and $a_{2}$ is made, then (7.2) determines uniquely $r_{1}$ and $p_{2}$. Then one obtains $q_{1}$ form $\mathcal{D}$ and also $q_{3}=\left(a_{1}-1\right) /\left(p_{2}-1\right)$.

Assume for contradiction that by interchanging $a_{1}$ and $a_{2}$ we get another set of solutions $\widetilde{r_{1}}, \widetilde{p_{2}}$ and so on. Then, by (7.2), $\widetilde{r_{1}}=a_{1}-p_{2}+1$ and $\widetilde{p_{2}}=a_{2}-r_{1}$. As $\widetilde{p_{2}} \in\left\{p_{2}, q_{1}\right\}$, there are two cases.

If $\widetilde{p_{2}}=q_{1}$, then substituting this in the expression of $\widetilde{p_{2}}$ and using (7.1) for $a_{2}$ produces $\left(q_{3}-2\right) r_{1}=-1$, whose left-hand side is non-negative, a contradiction.

On the other hand, if $\widetilde{p_{2}}=p_{2}$, then from the expression of $a_{3}$ in (7.1) (used for both sets of solutions) we obtain $\widetilde{r_{1}}=r_{1}$. Thus, again from (7.1), we obtain $a_{1}=a_{2}$ contradicting $\operatorname{gcd}\left(a_{1}, a_{2}\right)=1$.

\subsection{Two non-degenerate arms}

7.3.1 Assume that there are either at least two vertices which are not in the arm of $v_{1}$, or there is only one such vertex, namely, $v_{2}$. In the latter case, we also assume that $v_{2}$ is an ER-hand because the other case is treated in $\S 7.2$. Anyway, $v_{2}$ is also a hand, so we also preprocess its arm. We face two cases: either the two arms (of $v_{1}$ and $v_{2}$ ) cover all of the vertices of $G^{\circ}$ (this fact characterizes the family $\mathbf{1}_{2}$ ), or the arms contain all of the vertices but one, which should be a central vertex/face (this is the family $\boldsymbol{\Delta}_{2}$ ).

7.3.2 The case $\mathbf{1}_{2}$. If the arm of $v_{2}$ contains all of the vertices then we are in the situation of $\S 7.1 .5$, and we are done. Assume that this is not the case. Fix the coordinates $z_{i}$ so that the arm of $v_{i}$ is in the direction of $z_{i}(i=1,2)$. We select (arbitrarily) a common edge $\alpha=[(p, q, 0),(0,0, c)]$ of the two arms, and let $\triangle_{i}$ be the face adjacent to it in the direction $z_{i}$. In particular, $\triangle_{i}$ lies in the arm of $v_{i}$. Let $\overrightarrow{\mathbf{a}}^{(i)}$ be the normal vector of $\triangle_{i}$. We seek the coordinates of these vectors and the edge $\alpha$.

By preprocessing the arms, we have obtained the sets $\{c, p\}$ and $\{c, q\}$, and the first two coordinates of both $\overrightarrow{\mathbf{a}}^{(i)}$. By (A.3) one has $\overrightarrow{\mathbf{a}}^{(1)} \times \overrightarrow{\mathbf{a}}^{(2)}=(-p,-q, c)$, hence $c=a_{1}^{(1)} a_{2}^{(2)}-a_{2}^{(1)} a_{1}^{(2)}$. Hence, we recover $\alpha$. Moreover, by face value computation, $a_{3}^{(i)} c=p a_{1}^{(i)}+q a_{2}^{(i)}$, hence we get the normal vectors as well. The algorithm finishes with postprocessing the arms. 


\section{G. Braun AND A. NÉmethi}

7.3.3 The case $\boldsymbol{\Delta}_{2}$. Similarly as above, fix the coordinates $z_{i}$ so that the arm of $v_{i}$ is in the direction of $z_{i}(i=1,2)$. We wish to determine the central triangle $\triangle$ using Lemma 5.4.1, the notation of which we will use. Preprocessing the two non-degenerate arms, we have determined the sets $\left\{r_{2}, q_{3}\right\}$ and $\left\{p_{3}, r_{1}\right\}$, and the first two coordinates of $\overrightarrow{\mathbf{a}} \triangle$. The third coordinate of $\overrightarrow{\mathbf{a}} \triangle$ is the decoration of the leg adjacent to the vertex corresponding to $\triangle$, hence $\overrightarrow{\mathbf{a}} \triangle$ is known from $G^{o}$. For $1 \leqslant i \leqslant 3$ denote by $\nabla_{i}$ the face of $\Gamma_{+}$adjacent to $\triangle$ in the direction of the axis $z_{i}$. Then, by the notation of Lemma 4.4.7, we already know the coordinates $a_{1}^{(1)}$ and $a_{2}^{(2)}$ from preprocessing the arms. Furthermore, $\nabla_{3}$ is a non-compact face with $a_{3}^{(3)}=0$ by Lemma A.1.3. Therefore, (4.11) gives the face value $m_{\triangle}$. This, via (4.7) and Corollary 4.4.5, provides all of the face values, in particular the face value of $\nabla_{3}$ too. This is $p_{2} q_{1}$. As either $p_{2}$ or $q_{1}$ is 1 , we get the set $\left\{p_{2}, q_{1}\right\}$ as well. Hence, Lemma 5.4.1 determines $\triangle$ (up to a permutation of coordinates). Then postprocessing the arms recovers the Newton diagram.

\section{Appendix A}

\section{A.1 Some arithmetical properties of Newton boundaries}

Lemma A.1.1. Let $\triangle$ be a triangle whose vertices are lattice points. Let $\overrightarrow{\mathbf{a}}$ and $\overrightarrow{\mathbf{b}}$ be the vectors of two of its sides. Then

$$
\overrightarrow{\mathbf{a}} \times \overrightarrow{\mathbf{b}}= \pm g(\triangle) \overrightarrow{\mathbf{a}} \triangle
$$

In particular, if $\triangle$ is an empty triangle with vertices $\left(0, p_{2}, p_{3}\right),\left(q_{1}, 0, q_{3}\right)$ and $\left(r_{1}, r_{2}, 0\right)$, then

$$
\overrightarrow{\mathbf{a}}_{\triangle}=\left(p_{2} q_{3}+r_{2} p_{3}-r_{2} q_{3}, q_{3} r_{1}+p_{3} q_{1}-p_{3} r_{1}, r_{1} p_{2}+q_{1} r_{2}-q_{1} p_{2}\right) .
$$

Proof. By the additivity of $g(\triangle)$, we may assume that $\triangle$ is empty. In that case $\overrightarrow{\mathbf{a}}$ and $\overrightarrow{\mathbf{b}}$ can be completed to a base (see, e.g., [Oda88, p. 35]), hence $\overrightarrow{\mathbf{a}} \times \overrightarrow{\mathbf{b}}$ is primitive. The second part is a direct application. To verify the sign, note that the scalar product of both vectors in (A.2) with the vertices of the triangle are positive.

Lemma A.1.2. Let $\triangle$ and $\nabla$ be two adjacent lattice polygons.

(a) Then the vector $\overrightarrow{\mathbf{v}}$ of their common edge is, up to a sign:

$$
\overrightarrow{\mathbf{v}}= \pm \frac{t_{\triangle, \nabla}}{n_{\triangle, \nabla}} \overrightarrow{\mathbf{a}}_{\triangle} \times \overrightarrow{\mathbf{a}}_{\nabla}
$$

(b) Assume that $\triangle$ and $\nabla$ are adjacent faces of a Newton polytope, $\triangle$ is a triangle, and let $\overrightarrow{\mathbf{a}}$ be a vector from a point from their common edge to the third vertex of $\triangle$. Then

$$
n_{\triangle, \nabla}=\frac{t_{\triangle, \nabla}}{g(\triangle)}\left\langle\overrightarrow{\mathbf{a}}, \overrightarrow{\mathbf{a}}_{\nabla}\right\rangle
$$

(c) Let $\triangle$ be a triangle with vertices $\left(0, p_{2}, p_{3}\right),\left(q_{1}, 0, q_{3}\right)$, and $\left(q_{1}^{\prime}, 0, q_{3}^{\prime}\right)$ with $q_{1}^{\prime}<q_{1}$, situated on a compact face of a Newton boundary. Assume that $\triangle$ has no lattice points other than its vertices and possible internal lattice points on its side on the $z_{1} z_{3}$ plane. Then the following expressions are equal and integers:

$$
\frac{q_{1}-q_{1}^{\prime}}{t_{\triangle, \overrightarrow{\mathbf{e}_{\mathbf{2}}}}}=\frac{\left\langle\overrightarrow{\mathbf{a}} \triangle, \overrightarrow{\mathbf{e}_{3}}\right\rangle}{n_{\triangle, \overrightarrow{\mathbf{e}_{\mathbf{2}}}}} \in \mathbb{N}
$$

In fact,

$$
n_{\triangle, \overrightarrow{\mathbf{e}_{2}}}=p_{2}
$$

Proof. (a) The vector $\pm \overrightarrow{\mathbf{v}}$ is characterized by the fact that it is orthogonal to both normal vectors and it is $t_{\triangle, \nabla}$ times a primitive vector. The vector on the right-hand side of (A.3) has this property. 


\section{INVARIANTS OF NEWTON NON-DEGENERATE SURFACE SINGULARITIES}

For part (b), because $\overrightarrow{\mathbf{v}}$ is orthogonal to $\overrightarrow{\mathbf{a}}_{\nabla}$, (A.3) and (A.1) give

$$
\pm g(\triangle) \frac{n_{\triangle, \nabla}}{t_{\triangle, \nabla}} \overrightarrow{\mathbf{v}}= \pm g(\triangle)\left(\overrightarrow{\mathbf{a}}_{\triangle} \times \overrightarrow{\mathbf{a}}_{\nabla}\right)=(\overrightarrow{\mathbf{a}} \times \overrightarrow{\mathbf{v}}) \times \overrightarrow{\mathbf{a}}_{\nabla}=\left\langle\overrightarrow{\mathbf{a}}, \overrightarrow{\mathbf{a}}_{\nabla}\right\rangle \overrightarrow{\mathbf{v}}
$$

This gives (A.4) up to a sign. As the scalar product of the normal vector of a face assigns its minimum on the face (when restricted to the Newton boundary), the scalar product in (A.4) is positive, and hence both sides of (A.4) are positive.

For (A.5), we apply part (a) with $\overrightarrow{\mathbf{a}}_{\nabla}=\overrightarrow{\mathbf{e}_{\mathbf{2}}}$ and $\overrightarrow{\mathbf{v}}=\left(q_{1}-q_{1}^{\prime}, 0, q_{3}-q_{3}^{\prime}\right)$. First note that $\overrightarrow{\mathbf{v}}$ is $t_{\triangle, \overrightarrow{\mathbf{e}_{\mathbf{2}}}}$ times a primitive vector, hence $\left(q_{1}-q_{1}^{\prime}\right) / t_{\triangle, \overrightarrow{\mathbf{e}_{\mathbf{2}}}}=\left\langle\overrightarrow{\mathbf{v}} / t_{\triangle, \overrightarrow{\mathbf{e}_{2}}}, \overrightarrow{\mathbf{e}_{\mathbf{1}}}\right\rangle \in \mathbb{N}$.

On the other hand, taking scalar product of (A.3) with $\overrightarrow{\mathbf{e}_{1}}$, we obtain (A.5) up to a sign. As both expression are positive in (A.5), the sign is correct. The last equality is a special case of part (b) with $\nabla$ the $z_{1} z_{3}$ plane, because $g(\triangle)=t_{\triangle, \overrightarrow{\mathbf{e}_{2}}}$.

Recall (cf. Remark 2.1.5) that a non-compact face of $\Gamma$ (with (2.1)) either lies on a coordinate plane, or it has an edge of type $[(a, 0, c),(0,1, b)]$ and normal vector $(1, a, 0)$ with $a>0$.

Lemma A.1.3. Let an edge $A B=[(a, 0, c),(0,1, b)]$ lie on a compact face and on a non-compact one with normal vectors $\overrightarrow{\mathbf{a}}$ and $\overrightarrow{\mathbf{n}}:=(1, a, 0)$, respectively $(a>0)$. Then

$$
n_{\overrightarrow{\mathbf{n}}, \overrightarrow{\mathbf{a}}}=\left\langle\overrightarrow{\mathbf{a}}, \overrightarrow{\mathbf{e}_{\mathbf{3}}}\right\rangle \text {. }
$$

Assume that $C=(r, s, u)$ is a third vertex of the compact face, such that the triangle $\triangle_{A B C}$ is empty. Then the determinant is also

$$
n_{\overrightarrow{\mathbf{n}}, \overrightarrow{\mathbf{a}}}=\langle(r-a, s, u-c), \overrightarrow{\mathbf{n}}\rangle=r+(s-1) a .
$$

Proof. Let $\triangle$ be the empty triangle on the non-compact face with vertices $(a, 0, c),(0,1, b)$ and $(0,1, b+1)$, and let $\nabla$ denote the compact face. Then (A.4) with $\overrightarrow{\mathbf{a}}=\overrightarrow{\mathbf{e}_{\mathbf{3}}}$ yields (A.7). The other equation is again an application of (A.4). However, this time $\nabla$ is the non-compact face, and $\triangle$ is the triangle with vertices $(a, 0, c),(0,1, b)$, and $(r, s, u)$.

Lemma A.1.4. Let $\triangle$ and $\nabla$ be two adjacent triangular faces of a Newton diagram whose vertices lie on the coordinate planes containing the $z_{3}$ axis. Further, let us assume that $\triangle$ has an edge on the $z_{1} z_{3}$ plane, which contains all of the lattice points of the triangle except the third vertex. Let its determinant $n_{\triangle, \overrightarrow{\mathbf{e}_{2}}}$ be denoted by $n_{\triangle}$. Similarly, we suppose that $\nabla$ has an edge $\alpha$ either on the $z_{1} z_{3}$ plane or on the $z_{2} z_{3}$ plane containing all lattice points except the third vertex. Its determinant will be denoted by $n_{\nabla}$. Then

$$
\begin{aligned}
& \alpha \in z_{1} z_{3} \text { plane } \quad \Longleftrightarrow \quad n_{\triangle}=n_{\nabla} \mid n_{\triangle, \nabla}, \\
& \alpha \in z_{2} z_{3} \text { plane } \quad \Longleftrightarrow \quad \operatorname{gcd}\left(n_{\triangle}, n_{\nabla}, n_{\triangle, \nabla}\right)=1 .
\end{aligned}
$$

Proof. Let $\overrightarrow{\mathbf{v}}$ be the vector of the common edge of the triangles. Let $\overrightarrow{\mathbf{a}}$ be the primitive vector parallel to the edge of $\triangle$ lying on the $z_{1} z_{3}$ plane. Finally, let $\overrightarrow{\mathbf{c}}$ be the primitive vector parallel to $\alpha$. Now, (A.3) combined with (A.1) implies that $n_{\triangle, \nabla}$ equals the triple product $\overrightarrow{\mathbf{a}} \overrightarrow{\mathbf{v}} \overrightarrow{\mathbf{c}}$ (up to a sign).

If $\alpha$ lies on the $z_{1} z_{3}$ plane, $n_{\triangle}=n_{\nabla}=\left\langle\overrightarrow{\mathbf{v}}, \overrightarrow{\mathbf{e}_{\mathbf{2}}}\right\rangle$ by (A.6). As the second coordinates of $\overrightarrow{\mathbf{a}}$ and $\overrightarrow{\mathbf{c}}$ are 0 , the number $\left\langle\overrightarrow{\mathbf{v}}, \overrightarrow{\mathbf{e}_{\mathbf{2}}}\right\rangle$ divides the triple product. This proves the ' $\Rightarrow$ ' part of (A.9).

If $\alpha$ lies on the $z_{2} z_{3}$ plane, then $\left\langle\overrightarrow{\mathbf{a}}, \overrightarrow{\mathbf{e}_{\mathbf{2}}}\right\rangle=\left\langle\overrightarrow{\mathbf{c}}, \overrightarrow{\mathbf{e}_{\mathbf{1}}}\right\rangle=0$. Therefore, $n_{\triangle, \nabla}$, modulo the greatest common divisor $d$ of $n_{\triangle}=\left\langle\overrightarrow{\mathbf{v}}, \overrightarrow{\mathbf{e}_{\mathbf{2}}}\right\rangle$ and $n_{\nabla}=\left\langle\overrightarrow{\mathbf{v}}, \overrightarrow{\mathbf{e}_{\mathbf{1}}}\right\rangle$, is

$$
n_{\triangle, \nabla}=\overrightarrow{\mathbf{a}} \overrightarrow{\mathbf{v}} \overrightarrow{\mathbf{c}} \equiv-\left\langle\overrightarrow{\mathbf{a}}, \overrightarrow{\mathbf{e}_{\mathbf{1}}}\right\rangle \cdot\left\langle\overrightarrow{\mathbf{v}}, \overrightarrow{\mathbf{e}_{\mathbf{3}}}\right\rangle \cdot\left\langle\overrightarrow{\mathbf{c}}, \overrightarrow{\mathbf{e}_{2}}\right\rangle \quad(\bmod d)
$$

The three terms of the right-hand side are relative prime to $d$ because $\overrightarrow{\mathbf{a}} \triangle= \pm \overrightarrow{\mathbf{a}} \times \overrightarrow{\mathbf{v}}, \overrightarrow{\mathbf{a}}_{\nabla}=$ $\pm \overrightarrow{\mathbf{c}} \times \overrightarrow{\mathbf{v}}$, and $\overrightarrow{\mathbf{v}}$ are primitive. Hence, the ' $\Rightarrow$ ' part of (A.10) follows. We end the proof by noting that the right-hand sides of (A.9) and (A.10) are mutually exclusive. 


\section{G. Braun AND A. NÉmethi}

\section{A.2 The weighted homogeneous case (graphs with one node)}

Below $\mathcal{L}=\left(d_{1}, k_{1} ; \ldots ; d_{s}, k_{s}\right)$ means that the unique vertex of $G^{o}$ has $s$ leg-groups, the $i$ th group has size $k_{i} \geqslant 1$ and decoration $d_{i}>1$ (with $d_{i} \neq d_{j}$ for $i \neq j$, and $\sum_{i} k_{i} \geqslant 3$ ). The number $e$ is the orbifold Euler number. One has the following cases:

(1) $\mathcal{L}=(d, k)$,

equation: $z_{1}^{d}+z_{2}^{k-1} z_{3}+z_{2} z_{3}^{k-1}$

(2) $\mathcal{L}=(d, 2 ; D, 2)$,

equation: $z_{1}^{d} z_{3}+z_{2}^{2 D}+z_{3}^{2}$, equivalently $z_{1}^{2 d}+z_{2}^{D} z_{3}+z_{3}^{2}$,

(the equations are $\sim$-equivalent);

(3) $\mathcal{L}=(d, k ; D, 1), d \mid D$,

equation: $z_{1}^{k} z_{2}+z_{1} z_{2}^{(k-1) D / d+1}+z_{3}^{d}$;

(4) $\mathcal{L}=(d, k ; D, 1), \operatorname{gcd}(d, D)=1,-e d D=1$,

equation: $z_{1}^{d}+z_{2}^{(k-1) / D} z_{3}+z_{2} z_{3}^{k}$;

(5) $\mathcal{L}=(d, k ; D, 1), \operatorname{gcd}(d, D)=1,-e d D=k$,

equation: $z_{1}^{d} z_{2}+z_{2}^{(D+1)(k-1) / k} z_{3}+z_{3}^{k}$;

(6) $\mathcal{L}=(a, 2 ; b, 2 ; c, 2)$,

equation: $z_{1}^{2 a}+z_{2}^{2 b}+z_{3}^{2 c}$;

(7) $\mathcal{L}=(a, k ; b, 1 ; c, 1), a|b, a| c$,

equation: $z_{1}^{(b k) / a+1} z_{2}+z_{1} z_{2}^{(c k) / a+1}+z_{3}^{a}$;

(8) $\mathcal{L}=(a, k ; b, 1 ; c, 1), b \mid c$ and $k>1$,

equation: $z_{1}^{a} z_{2}+z_{2}^{c / b+1}+z_{3}^{k b}$

(9) $\mathcal{L}=(a, k ; b, 1 ; c, 1), a \mid b, a \nmid c,-e b c=1$,

equation: $z_{1}^{k c} z_{2}+z_{2}^{(b k) / a+1}+z_{3}^{a}$;

(10) $\mathcal{L}=(a, k ; b, 1 ; c, 1), a \mid b, a \nmid c, A:=-e b c>1$,

equation: $z_{1}^{(k c-1) / A+1} z_{2}+z_{2}^{A}+z_{1} z_{3}^{a}$;

(11) $\mathcal{L}=(a, k ; b, 1 ; c, 1)$, the numbers $a, b, c$ do not divide each other, and $-e a b c=k^{2}$, equation: $z_{1}^{a}+z_{2}^{k c}+z_{3}^{k b}$

(12) $\mathcal{L}=(a, k ; b, 1 ; c, 1)$, the numbers $a, b, c$ do not divide each other, $k=1$ and $A:=-e a b c>1$, equation: $z_{1}^{(A-b) / a} z_{2}+z_{2}^{(A-c) / b} z_{3}+z_{3}^{(A-a) / c} z_{1}$, or $z_{1}^{(A-b) / c} z_{2}+z_{2}^{(A-a) / b} z_{3}+z_{3}^{(A-c) / a} z_{1}$, (only one of the equations have integer exponents, and this equation gives the right diagram).

\section{REFERENCES}

AGV88 V. I. Arnol'd, S. M. Guseřn-Zade and A. N. Varchenko, Singularities of differentiable maps. Vol. II, Monographs in Mathematics, vol. 83 (Birkhäuser, Boston, MA, 1988); MR: 966191 (89g:58024).

BLDM07 E. A. Bartolo, I. Luengo, J. F. de Bobadilla and A. Melle-Hernández, Milnor number of weighted Iomdine singularities, Preprint (2007).

BS75 J. Briançon and J.-P. Speder, La trivialité topologique n'implique pas les conditions de Whitney, C. R. Acad. Sci. Paris Sér. A 280 (1975), 365-367; MR: 0425165 (54 \#13122).

Dan79 V. I. Danilov, Newton polyhedra and vanishing cohomology, Funktsional. Anal. i Prilozhen. 13 (1979), 32-47; MR: 541636 (80h:14001).

HR89 H. Hauser and R. Randell, Report on the problem session, in Singularities, Iowa City, IA, 1986, Contemporary Mathematics, vol. 90 (American Mathematical Society, Providence, RI, 1989), 119-134; MR: 1000597 (90c:32017). 


\section{INVARIANTS OF NEWTON NON-DEGENERATE SURFACE SINGULARITIES}

Kou76 A. G. Kouchnirenko, Polyèdres de Newton et nombres de Milnor, Invent. Math. 32 (1976), 1-31; MR: 0419433 (54 \#7454).

Lau83 H. B. Laufer, Weak simultaneous resolution for deformations of Gorenstein surface singularities, in Singularities, Part 2, Arcata, CA, 1981, Proceedings of Symposia in Pure Mathematics, vol. 40 (American Mathematical Society, Providence, RI, 1983), 1-29; MR: 713236 (84k:32030).

Lau87 H. B. Laufer, Strong simultaneous resolution for surface singularities, in Complex analytic singularities, Advanced Studies in Pure Mathematics, vol. 8 (North-Holland, Amsterdam, 1987), 207-214; MR: 894294 (88g:32024).

LMN05 I. Luengo-Velasco, A. Melle-Hernández and A. Némethi, Links and analytic invariants of superisolated singularities, J. Algebraic Geom. 14 (2005), 543-565; MR: 2129010 (2005m:32057).

MN05 R. Mendris and A. Némethi, The link of $\left\{f(x, y)+z^{n}=0\right\}$ and Zariski's conjecture, Compositio Math. 141 (2005), 502-524; MR: 2134278 (2006c:32039).

MT80 M. Merle and B. Teissier, Conditions d'adjonction, d'après Du Val, in Séminaire sur les singularités des surfaces, Palaiseau, 1976-1977, Lecture Notes in Mathematics, vol. 777, eds M. Demazure, H. C. Pinkham and B. Teissier (Springer, Berlin, 1980), 229-247; MR: 579026 (82d:14021).

Mum61 D. Mumford, The topology of normal singularities of an algebraic surface and a criterion for simplicity, Publ. Math. Inst. Hautes Études Sci. 9 (1961), 5-22; MR: 0153682 (27 \#3643).

Ném04 A. Némethi, Invariants of normal surface singularities, in Real and complex singularities, Contemporary Mathematics, vol. 354 (American Mathematical Society, Providence, RI, 2004), 161-208; MR: 2087811 (2005g:32040).

Neu81 W. D. Neumann, A calculus for plumbing applied to the topology of complex surface singularities and degenerating complex curves, Trans. Amer. Math. Soc. 268 (1981), 299-344; MR: 632532 (84a:32015).

Neu83 W. D. Neumann, Abelian covers of quasihomogeneous surface singularities, in Singularities, Part 2, Arcata, CA, 1981, Proceedings of Symposia in Pure Mathematics, vol. 40 (American Mathematical Society, Providence, RI, 1983), 233-243; MR: 713252 (85g:32018).

NN02 A. Némethi and L. I. Nicolaescu, Seiberg-Witten invariants and surface singularities, Geom. Topol. 6 (2002), 269-328; MR: 1914570 (2003i:14048).

NW02 W. D. Neumann and J. Wahl, Universal abelian covers of surface singularities, in Trends in singularities, Trends in Mathematics (Birkhäuser, Boston, MA, 2002), 181-190; MR: 1900786 (2003c:32028).

Oda88 T. Oda, Convex bodies and algebraic geometry, in An introduction to the theory of toric varieties, Ergebnisse der Mathmatik und ihrer Grenzgebiete (3), vol. 15 (Springer, Berlin, 1988); MR: 922894 (88m:14038).

Oka79 M. Oka, On the bifurcation of the multiplicity and topology of the Newton boundary, J. Math. Soc. Japan 31 (1979), 435-450; MR: 535090 (80h:32018).

Oka87 M. Oka, On the resolution of the hypersurface singularities, in Complex analytic singularities, Advanced Studies in Pure Mathematics, vol. 8 (North-Holland, Amsterdam, 1987), 405-436; MR: 894303 (88m:32023).

Oka89 M. Oka, On the weak simultaneous resolution of a negligible truncation of the Newton boundary, Singularities, Iowa City, IA, 1986, Contemporary Mathematics, vol. 90 (American Mathematical Society, Providence, RI, 1989), 199-210; MR: 1000603 (90h:32030).

Par99 A. Parusiński, Topological triviality of $\mu$-constant deformations of type $f(x)+\operatorname{tg}(x)$, Bull. London Math. Soc. 31 (1999), 686-692; MR: 1711027 (2001d:58048).

Pin77 H. Pinkham, Normal surface singularities with $C^{*}$ action, Math. Ann. 227 (1977), 183-193; MR: 0432636 (55 \#5623).

Sai88 M. Saito, Exponents and Newton polyhedra of isolated hypersurface singularities, Math. Ann. 281 (1988), 411-417; MR: 954149 (89h:32027).

Ste77 J. H. M. Steenbrink, Mixed Hodge structure on the vanishing cohomology, in Real and complex singularities, Proceedings of Ninth Nordic Summer School/NAVF Symp. Mathematics, Oslo, 1976 (Sijthoff and Noordhoff, Alphen aan den Rijn, 1977), 525-563; MR: 0485870 (58 \#5670). 


\section{INVARIANTS OF NEWTON NON-DEGENERATE SURFACE SINGULARITIES}

Ste85 J. H. M. Steenbrink, Semicontinuity of the singularity spectrum, Invent. Math. 79 (1985), 557-565; MR: 782235 (86h:32033).

Tro80 D. Trotman, Equisingularité et conditions de Whitney, Thèse d'Etat, Université de Paris-Sud, Orsay (1980).

Var76 A. N. Varchenko, Zeta-function of monodromy and Newton's diagram, Invent. Math. 37 (1976), 253-262; MR: 0424806 (54 \#12764).

Var82 A. N. Varchenko, The complex exponent of a singularity does not change along strata $\mu=$ const., Funct. Anal. Appl. 16 (1982), 1-9.

VK85 A. N. Varchenko and A. G. Khovanskiü, Asymptotic behavior of integrals over vanishing cycles and the Newton polyhedron, Dokl. Akad. Nauk SSSR 283 (1985), 521-525; MR: 800892 (87e:32012).

Wag83 P. Wagreich, The structure of quasihomogeneous singularities, in Singularities, Part 2, Arcata, CA, 1981, Proceedings of Symposia Pure Mathematics, vol. 40 (American Mathematical Society, Providence, RI, 1983), 593-611; MR: 713284 (85e:14051).

Wal99 C. T. C. Wall, Newton polytopes and non-degeneracy, J. reine angew. Math. 509 (1999), 1-19; MR: 1679164 (2000g:32032).

Zar71 O. Zariski, Some open questions in the theory of singularities, Bull. Amer. Math. Soc. 77 (1971), 481-491; MR: 0277533 (43 \#3266).

Gábor Braun braung@renyi.hu

Alfréd Rényi Institute of Mathematics, 1053 Budapest, Reáltanoda u. 13-15, Hungary

András Némethi nemethi@renyi.hu

Alfréd Rényi Institute of Mathematics, 1053 Budapest, Reáltanoda u. 13-15, Hungary 